\title{
RAMSEY'S THEOREM AND CONE AVOIDANCE
}

\author{
DAMIR D. DZHAFAROV AND CARL G. JOCKUSCH, JR.
}

\begin{abstract}
It was shown by Cholak, Jockusch, and Slaman that every computable 2-coloring of pairs admits an infinite low2 homogeneous set $H$. We answer a question of the same authors by showing that $H$ may be chosen to satisfy in addition $C \mathbb{Z}_{T} H$, where $C$ is a given noncomputable set. This is shown by analyzing a new and simplified proof of Seetapun's cone avoidance theorem for Ramsey's theorem. We then extend the result to show that every computable 2-coloring of pairs admits a pair of low2 infinite homogeneous sets whose degrees form a minimal pair.
\end{abstract}

\section{INTRODUCTION}

In this paper we show that the infinite homogeneous sets asserted to exist by Ramsey's theorem for pairs can be chosen to have low information content in a sense to be made precise below.

Definition 1.1. Fix an infinite set $S \subseteq \omega$ and $n, k \in \omega$.

(i) We denote by $[S]^{n}$ the collection of all sets $T \subseteq S$ of cardinality $n$.

(ii) A $k$-coloring $f$ of $[S]^{n}$ is a map $[S]^{n} \longrightarrow\{0,1, \ldots, k-1\}$. If $S=\omega$ and $n=2$, we call $f$ a $k$-coloring of pairs.

(iii) We call a $k$-coloring $f$ of $[S]^{2}$ stable if for each $s \in S$ there exist $i<k$ and $t_{0} \in \omega$ such that $f(\{s, t\})=i$ for all $t \in S$ with $t>t_{0}$.

(iv) A set $H \subseteq S$ is said to be homogeneous for a $k$-coloring $f$ of $[S]^{n}$ if $f$ is constant on $[H]^{n}$.

Ramsey's theorem for $k$-colorings of exponent $n$ (denoted $\mathrm{RT}_{k}^{n}$ ) asserts that for every $k$-coloring of $[\omega]^{n}$ there exists an infinite homogeneous set. The effective content and logical strength of $\mathrm{RT}_{k}^{n}$ has been studied in [6], [9], and [1], among other papers.

Definition 1.2. Given a sequence $C_{0}, C_{1}, \ldots$ of sets, we call a set $S$ cone avoiding (for this sequence) provided $C_{i} \mathbb{Z}_{T} S$ for all $i \in \omega$.

We begin by recalling the following two results, both answering questions of Jockusch [6], pages 274 and 277.

Theorem 1.3 (Seetapun [9], Theorem 2.1). Given a sequence $C_{0}, C_{1}, \ldots$ of noncomputable sets, every computable 2-coloring of pairs admits an infinite cone avoiding homogeneous set.

We are grateful to Denis Hirschfeldt for many insightful discussions throughout the preparation of this article, and in particular for first bringing to our attention the possibility of using our proof of Theorem 3.4 to prove Theorem 3.5. We thank Antonín Kučera and Robert Soare for helpful comments on some of the material in Section 2. We also thank Joseph Mileti for a careful reading of an early draft of this article. The second author was partially supported by NSFC Grand International Joint Project 'New Directions in Theory and Applications of Models of Computation', No. 60310213. 
This theorem was used in [9], Theorem 3.1, to show that $\mathrm{RT}_{2}^{2}$ is strictly weaker than $\mathrm{ACA}_{0}$ over $\mathrm{RCA}$. For information on the systems $\mathrm{ACA}_{0}$ and $\mathrm{RCA} \mathrm{A}_{0}$, and Reverse Mathematics in general, see Simpson [11].

Theorem 1.4 (Cholak, Jockusch, and Slaman [1], Theorem 3.1). Every computable 2-coloring of $[\omega]^{2}$ admits an infinite homogeneous set $H$ which is low $w_{2}$, i.e. $H^{\prime \prime} \leq_{T}$ $0^{\prime \prime}$.

Cholak, Jockusch, and Slaman gave two different proofs of the above theorem. The first proof (in Section 4 of [1]) featured control of the first jump of $H$. It established the stronger result ([1], Corollary 12.6) that every computable 2-coloring of pairs admits an infinite homogeneous set whose jump has degree at most $\mathbf{d}$, where $\mathbf{d}$ is a given degree $\gg \mathbf{0}^{\prime}$. (See Definition 2.6 and the subsequent discussion. Also see the remarks after the proof of Theorem 3.4 for an explanation of a missing point in the proof of this stronger result.) The other proof of Theorem 1.4 (in Section 5 of [1]) featured direct control of the second jump of $H$. It was adapted in [1], Theorem 10.2, to models of second order arithmetic to show that every $\Pi_{1}^{1}$ sentence provable from $R C A_{0}+\Sigma_{2}^{0}$-IND $+\mathrm{RT}_{2}^{2}$ is provable from just $R C A_{0}+\Sigma_{2}^{0}$-IND, where $\Sigma_{2}^{0}$-IND is the induction scheme for $\Sigma_{2}^{0}$ formulas. Seetapun and Slaman [9], page 580 , asked whether Theorem 1.3, for the special case where $C_{i} \equiv_{T} 0^{\prime}$ for all $i \in \omega$, could be effectivized to produce a non-high cone avoiding infinite homogeneous set. A positive answer (for arbitrary noncomputable $C_{i}$ ) was given by Cholak, Jockusch, and Slaman [1], Theorem 12.2. In view of Theorem 1.4, they in turn raised the following question:

Question 1.5 (Cholak, Jockusch, and Slaman [1], Question 13.11). Given a noncomputable set $C$, does every computable 2-coloring of pairs admit an infinite low $_{2}$ homogeneous set $H$ with $C \underline{\Sigma}_{T} H$ ?

In this paper, we present a new proof of Seetapun's theorem (Theorem 1.3) which is considerably more straightforward than both the original (cf. [9], Section 2) as well as the simplification of Hummel and Jockusch (cf. [3], Theorem 2.1). This proof is based in part on the method used by Cholak, Jockusch, and Slaman in [1], Section 4, to prove Theorem 1.4 and benefited from discussions with Steffen Lempp and a subsequent suggestion of Slaman for simplifying it. The proof proceeds by first reducing the result to the stable case, and this reduction is straightforward. The crucial new element in the proof is Lemma 4.7, which handles cone avoidance in the stable case. By keeping track of the effective content in this new proof, we are able to give an affirmative answer to Question 1.5. We extend this result to show that every computable 2-coloring of pairs admits a pair of $\mathrm{low}_{2}$ infinite homogeneous sets whose degrees form a minimal pair. The proof of this uses an extension of the technique of [1], Section 5, together with a new basis theorem for $\Pi_{1}^{0}$ classes. Along the way, we extend Theorem 2.5 of Jockusch and Stephan [5] to show that there is a minimal pair of low $_{2}$ cohesive degrees.

\section{BASIS THEOREMS}

The low basis theorem of Jockusch and Soare [7], Theorem 2.1, played a crucial role in the proof of Theorem 1.4. Hence, it is not surprising that a cone avoiding version of the Low Basis Theorem will play a central role in answering Question 1.5. The needed result is due to Linda Lawton (cf. [2], Theorem 4.1). 
Theorem 2.1 (Lawton). Let $T \subseteq 2^{<\omega}$ be an infinite computable tree, let $C_{0}, C_{1}, \ldots$ be a sequence of noncomputable sets, and let $C=\oplus_{i \in \omega} C_{i}$. Then $T$ has an infinite cone avoiding path $f$ such that $f^{\prime} \leq_{T} 0^{\prime} \oplus C$. Furthermore, an index for $f^{\prime}$ as a $\left(0^{\prime} \oplus C\right)$-computable set can be found $\left(0^{\prime} \oplus C\right)$-effectively from an index for $T$.

The theorem is proved by forcing with $\Pi_{1}^{0}$ classes, alternating between forcing the jump and applying the following lemma.

Lemma 2.2 (Lawton). Let $T \subseteq 2^{<\omega}$ be an infinite computable tree, let $C$ be any noncomputable set, and let $e \in \omega$. Then $T$ has an infinite computable subtree $\widetilde{T}$ such that $\Phi_{e}^{f} \neq C$ for all $f \in[\widetilde{T}]$, and an index for $\widetilde{T}$ can be found $\left(0^{\prime} \oplus C\right)$-effectively from $e$ and an index for $T$.

Proof. For each $n \in \omega$, define the computable tree

$$
U_{n}=\left\{\sigma \in T: \neg\left(\Phi_{e,|\sigma|}^{\sigma}(n) \downarrow=C(n)\right)\right\},
$$

and notice that $U_{n}$ must be infinite for some $n$. Indeed, if each $U_{n}$ were finite, then to compute $C(n)$ for a given $n$ we would need only to search for $m, k \in \omega$ such that $\Phi_{e,|\sigma|}^{\sigma}(n)=k$ for all $\sigma \in T$ of length $m$, from which we conclude that $C(n)=k$. This contradicts the assumption that $C$ is noncomputable. Clearly, an index for $U_{n}$ as a computable tree can be found $C$-effectively from $e$ and an index for $T$, and $0^{\prime}$ can determine whether or not $U_{n}$ is infinite. (For the latter, use that $U_{n} \subseteq 2^{<\omega}$.) Thus, $0^{\prime} \oplus C$ can find the least $n$ such that $U_{n}$ is infinite, and we set $\widetilde{T}=\bar{U}_{n}$.

Note, in particular, that if the sequence $C_{0}, C_{1}, \ldots$ is taken uniformly $\Delta_{2}^{0}$, Theorem 2.1 asserts the existence, in any non-empty $\Pi_{1}^{0}$ class of sets, of a low member which avoids the upper cone of each $C_{i}$. The following variation on this result, in the case where the $\Pi_{1}^{0}$ class in question contains no computable members, is included here for general interest. As a special case, we get that any non-empty $\Pi_{1}^{0}$ class of sets with no computable members contains a low set Turing incomparable with a given noncomputable low set.

Theorem 2.3. Let $T \subseteq 2^{<\omega}$ be an infinite computable tree with no computable paths, let $C_{0}, C_{1}, \ldots$ be a sequence of noncomputable sets, and let $D=\oplus_{i \in \omega} C_{i}^{\prime}$. Then $T$ contains an infinite path $f$ such that $\left.f\right|_{T} C_{i}$ for all $i \in \omega$, and $f^{\prime} \leq_{T} D$. Further, an index for $f^{\prime}$ as a D-computable function can be found D-effectively from an index for $T$.

We can obtain the theorem from the next lemma, by interspersing the construction used in its proof into that of Theorem 2.1.

Lemma 2.4. Let $T \subseteq 2^{<\omega}$ be an infinite computable tree with no computable paths, let $C$ be any set, and let $e \in \omega$. Then $T$ has an infinite computable subtree $\widetilde{T}$ such that $\Phi_{e}^{C} \neq f$ for all $f \in[\widetilde{T}]$, and an index for $\widetilde{T}$ can be found $C^{\prime}$-effectively from $e$ and an index for $T$.

Proof. Since $T \subseteq 2^{<\omega}$ is infinite and computable and has no computable paths, $T$ has at least two distinct paths, and thus there exist incompatible strings $\sigma_{1}$ and $\sigma_{2}$ such that each $\sigma_{i}$ is extendible to a path through $T$. Such $\sigma_{1}$ and $\sigma_{2}$ can be found $0^{\prime}$-effectively since the set of extendible nodes of $T$ is $0^{\prime}$-computable. Fix $n$ such that $\sigma_{1}(n) \downarrow \neq \sigma_{2}(n) \downarrow$. Use $C^{\prime}$ to determine whether $\Phi_{e}^{C}(n) \downarrow$. If not, let $\widetilde{T}=T$. If so, choose $j \in\{1,2\}$ such that $\Phi_{e}^{C}(n) \downarrow \neq \sigma_{j}(n) \downarrow$, and let $\widetilde{T}$ be the set of all strings $\sigma \in T$ which are compatible with $\sigma_{j}$. 
Finally, we present the following "minimal pair basis theorem" which will be central to our work in Section 6. Recall that two degrees $\mathbf{a}$ and $\mathbf{b}$ are said to form a minimal pair if $\mathbf{a} \cap \mathbf{b}=\mathbf{0}$ (we do not require that $\mathbf{a}$ and $\mathbf{b}$ be non-zero).

Theorem 2.5. Let $T \subseteq 2^{<\omega}$ be an infinite computable tree, and let $B$ be any set. Then $T$ has an infinite path $f$ with $f^{\prime \prime} \leq_{T} 0^{\prime \prime} \oplus B^{\prime}$ whose degree makes a minimal pair with that of $B$. Furthermore, an index for $f^{\prime \prime}$ as a $\left(0^{\prime \prime} \oplus B^{\prime}\right)$-computable set can be found $\left(0^{\prime \prime} \oplus B^{\prime}\right)$-effectively from an index for $T$.

Proof. We force with $\Pi_{1}^{0}$ classes. Set $T_{0}=T$ and suppose that for some $e \geq 0$, $T_{e} \subseteq 2^{<\omega}$ is an infinite computable tree of known index. For each $n \in \omega$, define

$$
U_{n}=\left\{\sigma \in T_{e}: \Phi_{e,|\sigma|}^{\sigma}(n) \uparrow\right\}
$$

the index of which as a computable tree can be found effectively from an index for $T_{e}$. First, ask $0^{\prime \prime}$ whether $U_{n}$ is infinite for some $n$, and if so, search $0^{\prime}$-effectively for the least such $n$ and set $T_{e+1}=U_{n}$. In this case, $\Phi_{e}^{f}$ is not total for any $f \in\left[T_{e+1}\right]$. Otherwise, we know that $\Phi_{e}^{f}$ is total for all $f \in\left[T_{e}\right]$. In this case, we ask $0^{\prime \prime}$ if there exist extendible nodes $\sigma, \tau \in T_{e}$ and an $n \in \omega$ such that $\Phi_{e,|\sigma|}^{\sigma}(n) \downarrow \neq \Phi_{e,|\tau|}^{\tau}(n) \downarrow$. If there do, find the least such $\sigma, \tau$, and $n$ in some fixed effective listing and inquire of $B^{\prime}$ whether $\Phi_{e}^{B}(n)$ converges. If it does, let $T_{e+1}$ consist of all nodes in $T_{e}$ comparable with $\rho$, where $\rho$ is the lexicographically smaller of $\sigma$ and $\tau$ such that $\Phi_{e}^{\rho}(n) \neq \Phi_{e}^{B}(n)$. Clearly $\Phi_{e}^{f} \neq \Phi_{e}^{B}$ for all $f \in\left[T_{e+1}\right]$ in this case. If $\Phi_{e}^{B}(n)$ diverges, or if there do not exist any such $\sigma, \tau$, and $n$, set $T_{e+1}=T_{e}$. Suppose now that $\Phi_{e}^{B}$ and $\Phi_{e}^{f}$ are equal and total, where $f \in\left[T_{e+1}\right]$. Then each $U_{n}$ is finite and $\sigma, \tau$ and $n$ as above did not exist. Given $n, \Phi_{e}^{B}(n)$ can now be computed as follows. First, search for the least $k$ such that $\Phi_{e,|\sigma|}^{\sigma}(n) \downarrow$ for all $\sigma \in T$ of length $k$, and then search for the least $\ell \geq k$ and $m \in \omega$ such that $\Phi_{e,|\sigma|}^{\sigma}(n)=m$ for all $\sigma \in T$ of length $k$ having an extension on $T$ of length $\ell$. The second search succeeds because for any extendible $\sigma$ on $T$ of length $k$ it must be that $\Phi_{e,|\sigma|}^{\sigma}(n)=\Phi_{e}^{B}(n)$, so $m$ must be equal to $\Phi_{e}^{B}(n)$.

As usual, we iterate the construction to build a sequence $2^{<\omega}=T_{0} \supseteq T_{1} \supseteq \cdots$ of infinite computable trees, and we take $f \in \bigcap_{e \in \omega} T_{e}$. Since the construction was $\left(0^{\prime \prime} \oplus B^{\prime}\right)$-effective, and since it decided for each $e$ the totality of $\Phi_{e}^{f}$, it follows that $f^{\prime \prime} \leq_{T} 0^{\prime \prime} \oplus B^{\prime}$.

We will apply this result when $B$ satisfies $B^{\prime} \leq_{T} 0^{\prime \prime}$, in which case of course the path $f$ of the previous theorem is $\operatorname{low}_{2}$.

We conclude this section with the following definition due to Simpson [10], page 648:

Definition 2.6. Let $\mathbf{a}$ and $\mathbf{b}$ be Turing degrees. Then we write $\mathbf{a} \gg \mathbf{b}$ to mean that every infinite b-computable tree $T \subseteq 2^{<\omega}$ has an a-computable path.

It is known that $\mathbf{a} \gg \mathbf{b}$ if and only if every $\mathbf{b}$-computable $\{0,1\}$-valued partial function has an a-computable total extension. From this it follows that there is a nonempty $\Pi_{1}^{0}$ class $\mathscr{P}$ such that every element of $\mathscr{P}$ has degree $\gg \mathbf{0}$. Namely, consider the class $\mathscr{P}$ of all $\{0,1\}$-valued (total) functions $g$ such that $g(\langle e, i\rangle)=$ $\Phi_{e}(i)$ whenever $\Phi_{e}(i) \downarrow \leq 1$. The low basis theorem applied to $\mathscr{P}$ consequently implies that there exists a low degree $\mathbf{a} \gg \mathbf{0}$. Theorem 2.1, on the other hand, gives for each sequence $C_{0}, C_{1}, \ldots$ of noncomputable sets the existence of a cone avoiding degree $\mathbf{a} \gg \mathbf{0}$ whose jump is below $\mathbf{0}^{\prime} \cup \operatorname{deg}\left(\oplus_{i \in \omega} C_{i}\right)$. And similarly Theorem 2.5 
implies that for each degree $\mathbf{b}$ there is a degree $\mathbf{a} \gg \mathbf{0}$ with $\mathbf{a}^{\prime \prime} \leq \mathbf{0}^{\prime \prime} \cup \mathbf{b}^{\prime}$ and $(\mathbf{a}, \mathbf{b})$ a minimal pair.

\section{Cone Avoidance And First Jump Control}

Recall that, given a sequence $\vec{R}=\left\{R_{0}, R_{1}, \ldots\right\}$ of sets, an infinite set $S$ is called $\vec{R}$-cohesive if for each $i \in \omega$ either $S \subseteq^{*} R_{i}$ or $S \subseteq^{*} \overline{R_{i}}$. If $\vec{R}$ contains exactly the computable sets then an $\vec{R}$-cohesive set $S$ is called $r$-cohesive, while if $\vec{R}$ contains exactly the computably enumerable sets then $S$ is called cohesive. It was shown by Jockusch and Stephan in [5], Corollary 2.4, that the r-cohesive and cohesive degrees coincide. (A degree is cohesive if it contains a cohesive set and $r$-cohesive if it contains an r-cohesive set.)

Theorem 1.4 was proved by using a low 2 r-cohesive set to reduce it to the stable case, and then proving the stable case by transforming it to a statement about $\Delta_{2}^{0}$ sets. We proceed analogously here, but adding cone avoidance. We state two key lemmas, delaying their proofs until Section 5 below, and derive from them our main results. For the remainder of this section, let a degree $\mathbf{d} \gg \mathbf{0}^{\prime}$ be fixed. Additionally, except where otherwise stated, let $C_{0}, C_{1}, \ldots$ be a fixed sequence of noncomputable sets, and let $C=\oplus_{i \in \omega} C_{i}$. "Cone avoiding" below refers to this sequence.

Lemma 3.1. There exists a cone avoiding $r$-cohesive set $X$, and if $C \leq_{T} 0^{\prime}$ then $X$ can be chosen with jump of degree at most $\mathbf{d}$.

Lemma 3.2. (i) For $k \geq 2$, if $A_{1}, \ldots, A_{k}$ is a sequence of disjoint sets whose union is $\omega$, then for some $i \leq k, A_{i}$ contains an infinite cone avoiding subset.

(ii) If $A_{1}, \ldots, A_{k}, C \leq_{T} 0^{\prime}$, then for some $i \leq k, A_{i}$ contains an infinite cone avoiding subset with jump of degree at most $\mathbf{d}$.

We will, in addition, need the following well-known result:

Lemma 3.3 (Cholak, Jockusch, and Slaman [1], page 9). (i) The restriction of any computable $k$-coloring of pairs to an r-cohesive set is stable.

(ii) If $f$ is a computable stable $k$-coloring of pairs, then there exist pairwise disjoint $\Delta_{2}^{0}$ sets $A_{1}, \ldots, A_{k}$ covering $\omega$, any infinite subset of any of which computes an infinite homogeneous set for $f$.

For clarity, we now restate Seetapun's theorem, and show how it follows from Lemmas 3.1 and 3.2. The effectiveness of Lemmas 3.1 and 3.2 allows us to add an effectiveness result here for the case where the sequence $C_{0}, C_{1}, \ldots$ is uniformly $\Delta_{2}^{0}$. The latter is already a partial answer to Question 1.5.

Theorem 3.4. (i) (Seetapun [9], Theorem 2.1) Every computable 2-coloring of pairs admits an infinite cone avoiding homogeneous set $H$.

(ii) If $C \leq_{T} 0^{\prime}$, then $H$ can be chosen so that $\operatorname{deg}(H)^{\prime} \leq \mathbf{d}$.

Proof. (i) Let $f$ be a computable 2-coloring of pairs. By Lemma 3.1, fix a cone avoiding r-cohesive set $X$, and let $p: \omega \longrightarrow X$ be its principal function. Define an $X$-computable 2-coloring $g$ of pairs by $g(\{s, t\})=f(\{p(s), p(t)\})$, for all $\{s, t\} \in[\omega]^{2}$, which must be stable by Lemma 3.3(i). By part (ii) of the same lemma, relativized to $X$, there exists a $\Delta_{2}^{0, X}$ set $A$, such that for any infinite subset $S$ of $A$ or $\bar{A}, X \oplus S$ computes an infinite homogeneous set for $f$. On the other hand, by Lemma 3.2(i), relativized to $X$, there is an infinite subset $S$ of either $A$ 
of $\bar{A}$ whose join with $X$ is cone avoiding. Hence, $g$ admits an infinite homogeneous set $\widetilde{H} \leq_{T} X \oplus S$, and so by definition of $g, H=p(\widetilde{H})$ is homogeneous for $f$. Now we have

implying that $H$ is cone avoiding.

$$
H \leq_{T} X \oplus \widetilde{H} \leq_{T} X \oplus S,
$$

(ii) Assume $C \leq_{T} 0^{\prime}$. By Theorem 6.5 of Simpson [10], there exists a degree e satisfying $\mathbf{d} \gg \mathbf{e} \gg \mathbf{0}^{\prime}$, so by Lemma 3.1 we may choose $X$ to satisfy $\operatorname{deg}(X)^{\prime} \leq \mathbf{e}$. In particular, $\mathbf{d} \gg \operatorname{deg}(X)^{\prime}$, and so we may use part (ii) of Lemma 3.2 above, relativized to $X$, in place of part (i) to find $S$ with $X \oplus S$ not only cone avoiding but also with jump of degree at most d. Defining $H$ as before, we thus have $\operatorname{deg}(H)^{\prime} \leq \operatorname{deg}(X \oplus S)^{\prime} \leq \mathbf{d}$, as desired.

We remark that using density of the degrees under $\gg$ in the proof of part (ii) above is also necessary to obtain Theorem 12.6 of [1] mentioned in the introduction, and is missing from the exposition in [1]. This was pointed out by Joseph Mileti.

An affirmative answer to Question 1.5 now easily follows.

Theorem 3.5. Let $C$ be noncomputable. Every computable 2-coloring $f$ of pairs admits an infinite low homogeneous set $H$ such that $C \mathbb{Z}_{T} H$.

Proof. We consider several cases:

Case 1: $C \not_{T} 0^{\prime \prime}$. By Theorem 1.4, $f$ admits an infinite $\operatorname{low}_{2}$ homogeneous set $H$, meaning $C$ cannot be computable in $H^{\prime \prime}$, and hence also not in $H$.

Case 2: $C \leq_{T} 0^{\prime}$. Fix a satisfying $\mathbf{a} \gg \mathbf{0}^{\prime}$ and $\mathbf{a}^{\prime} \leq \mathbf{0}^{\prime \prime}$, as in the discussion at the end of Section 2. Now the desired result follows by Theorem 3.4(ii) using a in place of $\mathbf{d}$.

Case 3: $C \not_{T} 0^{\prime}$ and $C \leq_{T} 0^{\prime \prime}$. By Lemma 2.1, relativized to $\mathbf{0}^{\prime}$, there exists a cone avoiding degree $\mathbf{a} \gg \mathbf{0}^{\prime}$ with $\mathbf{a}^{\prime} \leq \mathbf{0}^{\prime \prime} \cup \operatorname{deg}(C)=\mathbf{0}^{\prime \prime}$. By Theorem 1.4 (or rather, by the extension of this theorem mentioned following its statement above), $f$ admits an infinite homogeneous set $H$ such that $\operatorname{deg}(H)^{\prime} \leq \mathbf{a}$, so $H$ is $\operatorname{low}_{2}$. And since $\operatorname{deg}(C) \not \mathbf{a}$, it follows that $C$ is not computable in $H$.

We do not know whether Theorem 3.5 can be strengthened to assert that for every $C>_{T} 0$ every computable 2 -coloring of pairs admits an infinite homogeneous set $H \Varangle_{T} C$ with $\operatorname{deg}\left(H^{\prime}\right) \leq \mathbf{d}$. That is, we do not know whether the condition in Theorem 3.4(ii) that $C$ be $\Delta_{2}^{0}$ can be omitted in the situation where "cone avoiding" means simply $H \nsupseteq_{T} C$.

\section{Strategies for Meeting Requirements}

Lemmas 3.1 and 3.2 will be proved using Mathias forcing with suitable restrictions on the infinite part of the conditions. In this section we show that each requirement for these lemmas is dense for the relevant version of Mathias forcing, and we calculate the oracle needed to find the extensions needed to witness this density. We then complete the proofs of these lemmas in the next section. Throughout this section, we assume fixed a degree $\mathbf{d} \gg \mathbf{0}^{\prime}$. Lemma 4.4 below is a variation on the proof in Section 4.1 of [1] of Theorem 2.5 of Jockusch and Stephan [5]. Lemmas 4.6, 4.7, and 4.8 are all variations of Lemma 4.6 of Cholak, Jockusch, and Slaman [1]. The last of these was suggested to us by Denis Hirschfeldt.

We begin by recalling and fixing some terminology. 
Definition 4.1. (i) A (Mathias) precondition is a pair of sets $(D, L)$ with $D$ finite and $\max (D)<\min (L)$. A (Mathias) condition is a precondition $(D, L)$ such that $L$ is infinite.

(ii) A set $G$ satisfies a precondition $(D, L)$ if $D \subseteq G \subseteq D \cup L$.

(iii) A precondition $(\widetilde{D}, \widetilde{L})$ extends a precondition $(D, L)$, written $(\widetilde{D}, \widetilde{L}) \prec(D, L)$ if $\widetilde{L} \subseteq L$ and $\widetilde{D}$ satisfies $(D, L)$. We call such an extension a finite extension if $L-\widetilde{L}$ is finite.

(iv) We say a precondition $(D, L)$ is cone avoiding, computable, or low if $L$ is, respectively, cone avoiding, computable, or low. If $(D, L)$ is computable or low, an index for this precondition is a pair of numbers $(d, \ell)$ such that $d$ is the canonical index of $D$ and $\ell$ is, respectively, a $\Delta_{1}^{0}$ index for $L$ or a $\Delta_{1}^{0, K}$ index for $L^{\prime}$.

Definition 4.2. Fix $e \in \omega$ and a precondition $(D, L)$.

(i) We say $(D, L)$ forces agreement on $e$ if there is no $x \in \omega$, and there are no finite sets $F_{0}, F_{1}$ satisfying $(D, L)$ such that $\Phi_{e}^{F_{0}}(x) \downarrow \neq \Phi_{e}^{F_{1}}(x) \downarrow$.

(ii) We say $(D, L)$ forces e out of the jump if there is no finite set $F$ satisfying $(D, L)$ such that $e \in F^{\prime}$.

\subsection{Strategies for Proving Lemma 3.1.}

Lemma 4.3 (Cholak, Jockusch, and Slaman [1], Lemma 4.4). Let $R$ be any computable set, $(D, L)$ a computable condition, and $e \in \omega$.

(i) There is a computable extension of $(D, L)$ which is satisfied only by sets $G$ with $|G| \geq e$, and an index for it can be found effectively from e and an index for $(D, L)$.

(ii) There is a computable extension of $(D, L)$ which is satisfied only by sets $G$ with $G \subseteq^{*} R$ or $G \subseteq^{*} \bar{R}$, and an index for it can be found $\mathbf{d}$-effectively from e and an index for $(D, L)$.

(iii) There is a computable extension of $(D, L)$ such that $G^{\prime}(e)$ is the same for all sets $G$ satisfying it, and an index for it can be found $0^{\prime}$-effectively from $e$ and an index for $(D, L)$.

Lemma 4.4. Let $C$ be any set, $(D, L)$ a condition, and $e \in \omega$. Then there is a finite extension of $(D, L)$ such that, for all sets $G$ satisfying it, either $\Phi_{e}^{G} \neq C$, or $\Phi_{e}^{G}$ is not total, or else $\Phi_{e}^{G}$ is L-computable. Furthermore, there is a fixed uniform procedure for finding canonical indices of finite sets $H_{0}, H_{1}$ from e, the canonical index of $D$, and an oracle for $L^{\prime} \oplus C$ such that the desired finite extension of $(D, L)$ has the form $\left(D \cup H_{0}, L \backslash H_{1}\right)$.

Proof. We consider two cases, the distinction between which is uniformly $L^{\prime}$-effective in $e$ and the canonical index of $D$.

Case 1: $(D, L)$ does not force agreement on $e$. Then we search, $L^{\prime}$-effectively from $e$ and the canonical index of $D$, for $x \in \omega$, and for finite sets $F_{0}, F_{1}$ satisfying $(D, L)$ such that $\Phi_{e}^{F_{0}}(x) \downarrow \neq \Phi_{e}^{F_{1}}(x) \downarrow$. One of the two computations, say $\Phi_{e}^{F_{j}}(x)$, must differ from $C(x)$, and we can, uniformly $C^{\prime}$-effectively from $i, x, F_{0}$, and $F_{1}$, find such a $j$. We then let $\widetilde{D}=F_{j}$, and let $\widetilde{L}$ consist of the elements of $L$ greater than $\max \left(F_{j}\right)$ and use $\left(\Phi_{e}^{F_{j}}(x)\right)$. Any set $G$ satisfying $(\widetilde{D}, \widetilde{L})$ will agree with $F_{j}$ below $\operatorname{use}\left(\Phi_{e}^{F_{j}}(x)\right)$ and hence will satisfy $\Phi_{e}^{G}(x) \downarrow=\Phi_{e}^{F_{j}}(x) \downarrow \neq C(x)$.

Case 2: $(D, L)$ forces agreement on e. We then set $(\widetilde{D}, \widetilde{L})=(D, L)$, and claim that if $G$ satisfies $(\widetilde{D}, \widetilde{L})$ then either $\Phi_{e}^{G}$ is not total or else it is $L$-computable. Indeed, 
if $\Phi_{e}^{G}$ is total, then to compute its value on input $x$ we have only to $L$-effectively search through the canonical indices of finite sets satisfying $(D, L)$ until we find one, call it $F$, such that $\Phi_{e}^{F}(x) \downarrow$. Such an $F$ clearly exists since, for example, any sufficiently long initial segment of $G$ will do, and we have $\Phi_{e}^{F}(x)=\Phi_{e}^{G}(x)$ for any such $F$, else the previous case would have applied.

In either case, $(\widetilde{D}, \widetilde{L})$ is a finite extension of $(D, L)$ with the desired properties, and the desired uniformity follows easily from the construction.

4.2. Strategies for Proving Lemma 3.2. Let $C_{0}, C_{1}, \ldots$ be a sequence of noncomputable sets, and let $C=\oplus_{i \in \omega} C_{i}$.

Lemma 4.5 (Cholak, Jockusch, and Slaman [1], Lemma 4.6). Let $A$ be any set, $(D, L)$ a condition with $L \cap A$ and $L \cap \bar{A}$ infinite, and $e, i \in \omega$. There is a finite extension $(\widetilde{D}, \widetilde{L})$ of $(D, L)$ such that, for all sets $G$ satisfying this extension, $\mid G \cap$ $A \mid \geq e$ and $|G \cap \bar{A}| \geq i$ (so in particular, $(\widetilde{D}, \widetilde{L})$ is low or cone avoiding if $(D, L)$ is). Moreover, an index for this extension can be found $\left(0^{\prime} \oplus A\right)$-effectively from $e$, $i$, and an index for $(D, L)$.

The following lemma adds cone avoidance to Lemma 4.6 of [1].

Lemma 4.6. Let $A$ be any set, $(D, L)$ a cone avoiding condition, and $e, i \in \omega$. There is a cone avoiding extension $(\widetilde{D}, \widetilde{L})$ of $(D, L)$ such that either $(G \cap A)^{\prime}(e)$ is the same for all sets $G$ satisfying this extension, or $(G \cap \bar{A})^{\prime}(i)$ is the same for all such $G$. If, additionally, $\mathbf{d}$ is a degree with $\mathbf{d} \gg \mathbf{0}^{\prime}, A, C \leq_{T} 0^{\prime}$, and $(D, L)$ is low, then $(\widetilde{D}, \widetilde{L})$ can be chosen low, and its index can be found $\mathbf{d}$-effectively from $e, i$, and an index for $(D, L)$. Furthermore, d can compute whether $(\widetilde{D}, \widetilde{L})$ forces the value of $(G \cap A)^{\prime}(e)$ or $(G \cap \bar{A})^{\prime}(i)$ and what value is forced.

Proof. Define the $\Pi_{1}^{0, L}$ class $\mathscr{P}$ to consist of all (possibly finite) sets $S \subseteq L$ such that $(D \cap A, S)$ forces $e$ out of the jump and $(D \cap \bar{A}, L-S)$ forces $i$ out of the jump. We consider two cases.

Case 1: $\mathscr{P} \neq \varnothing$. By Theorem 2.1, relativized to $L$, there exists an $S \in P$ such that $L \oplus S$ is cone avoiding, and hence the same is true of $S$ and $L-S$. Fix such an $S$. At least one of the following subcases must apply since $L$ is infinite.

Subcase 1a: $S$ is infinite. We then set $(\widetilde{D}, \widetilde{L})=(D, S)$, and observe that if $G$ is any set satisfying $(\widetilde{D}, \widetilde{L})$, then $G \cap A$ satisfies $(D \cap A, S)$. Hence, $e \notin(G \cap A)^{\prime}$, else any initial segment $F$ of $G$ sufficiently long that $D \cap A \subseteq F \cap A$ and $\Phi_{e}^{F \cap A}(e) \downarrow$ would contradict that $(D \cap A, S)$ forces $e$ out of the jump.

Subcase 1b: $L-S$ is infinite. We set $(\widetilde{D}, \widetilde{L})=(D, L-S)$, and it follows by a similar argument that $i \notin(G \cap \bar{A})^{\prime}$ for all $G$ satisfying $(\widetilde{D}, \widetilde{L})$.

Case 2: $\mathscr{P}=\varnothing$. Then in particular $L \cap A \notin \mathscr{P}$, so at least one of the following subcases must hold:

Subcase 2a: Some finite set $F$ with $\Phi_{e}^{F}(e) \downarrow$ satisfies $(D \cap A, L \cap A)$. We let $\widetilde{D}=D \cup F$ and let $\widetilde{L}$ consist of those elements of $L$ greater than $\max (\widetilde{D})$ and use $\left(\Phi_{e}^{F}(e)\right)$. Since $\widetilde{L}={ }^{*} L$, it follows that $\widetilde{L}$ is cone avoiding. Furthermore, for any set $G$ satisfying $(\widetilde{D}, \widetilde{L}), G \cap A$ and $F$ agree below use $\left(\Phi_{e}^{F}(e)\right)$. It follows that $\Phi_{e}^{G \cap A}(e) \downarrow=\Phi_{e}^{F}(e)$, and hence $e \in(G \cap A)^{\prime}$. 
Subcase 2b: Some finite set $F$ with $\Phi_{i}^{F}(i) \downarrow$ satisfies $(D \cap \bar{A}, L-A)$. We let $\widetilde{D}=D \cup F$ and let $\widetilde{L}$ consist of those elements of $L$ greater than $\max (\widetilde{D})$ and use $\left(\Phi_{i}^{F}(i)\right)$. It follows analogously that $i \in(G \cap \bar{A})^{\prime}$.

In either case, $(\widetilde{D}, \widetilde{L})$ extends $(D, L)$ and clearly has the desired properties.

Now assume that $A$ and $C$ are computable in $0^{\prime}$, and that $(D, L)$ is low. Notice that an index for $\mathscr{P}$ as a $\Pi_{1}^{0, L}$ class can be found $L^{\prime}$-effectively, hence $0^{\prime}$-effectively, from $e, i$, and canonical indices for $D \cap A$ and $D \cap \bar{A}$, hence, since $A$ is $\Delta_{2}^{0}, 0^{\prime}$ effectively from an index for $(D, L)$. By the effectiveness of Theorem 2.1 relativized to $L$, we can, in Case 1 above, $\left(0^{\prime} \oplus C\right)$-effectively, hence $0^{\prime}$-effectively, from an index for $\mathscr{P}$, find $S$ and a $\Delta_{1}^{0, K}$ index for $(L \oplus S)^{\prime}$ so as to additionally satisfy

$$
(L \oplus S)^{\prime} \leq_{T} L^{\prime} \oplus C \leq_{T} 0^{\prime} \oplus C \equiv_{T} 0^{\prime} .
$$

It follows that $S$ and $L-S$ are both low, and hence that the extension $(\widetilde{D}, \widetilde{L})$ is, in every case considered above, not only cone avoiding but also low.

The distinction between Cases 1 and 2 is $L^{\prime}$-effective from an index for $\mathscr{P}$, and hence $0^{\prime}$-effective.

We now argue, as in [1], Lemma 4.6, that one can $\mathbf{d}$-effectively choose a true subcase (1a or 1b) of Case 1. Consider the two statements " $S$ is infinite" and " $L-S$ is infinite". These are $\Pi_{2}^{0, L \oplus S}$ statements, hence $\Pi_{2}^{0}$ statements since $L \oplus S$ is low, and indices for them as such can be found effectively from a $\Delta_{1}^{0, K}$ index for $(L \oplus S)^{\prime}$, an index for $(D, L)$, and an index for $\mathscr{P}$. Since at least one of the statements is true, it follows by choice of $\mathbf{d} \gg \mathbf{0}^{\prime}$ that we can $\mathbf{d}$-effectively choose one which is true. (This is true because there is a partial function $\psi$ computable in $0^{\prime}$ which given indices $a, b$ of $\Sigma_{2}^{0}$ statements at least one of which is true outputs an index ( $a$ or $b$ ) of one which is true, and $\mathbf{d}$ can compute a total function which extends $\psi$.)

Since $A$ is $\Delta_{2}^{0}$, the distinction between Subcases $2 \mathrm{a}$ and $2 \mathrm{~b}$ amounts to $0^{\prime}$ effectively searching through finite sets satisfying $(D \cap A, L \cap A)$ and $(D \cap \bar{A}, L-A)$ until we find one, call it $F$, such that either $\Phi_{e}^{F}(e) \downarrow$ or $\Phi_{i}^{F}(i) \downarrow$.

We conclude that, in any case, an index for $(\widetilde{D}, \widetilde{L})$ can be found d-effectively from $e, i$, and an index for $(D, L)$, as desired. Furthermore, we can d-effectively find a statement in the list $e \in(G \cap A)^{\prime}, e \notin(G \cap A)^{\prime}, i \in(G \cap \bar{A})^{\prime}, i \notin(G \cap \bar{A})^{\prime}$ which is true of all $G$ satisfying $(\widetilde{D}, \widetilde{L})$.

Lemma 4.7. Let $A$ be any set, $(D, L)$ a cone avoiding condition, and e, $i, s, t \in \omega$. There is a cone avoiding extension $(\widetilde{D}, \widetilde{L})$ of $(D, L)$ such that, for all sets $G$ satisfying this extension, either $\Phi_{e}^{G \cap A} \neq C_{s}$ or $\Phi_{i}^{G \cap \bar{A}} \neq C_{t}$. If, additionally, $A, C \leq_{T} 0^{\prime}$ and $(D, L)$ is low, then $(\widetilde{D}, \widetilde{L})$ can be chosen low, and its index can be found $\mathbf{d}$ effectively from $e, i$, and an index for $(D, L)$.

Proof. Define $\mathscr{P}$ to be the class of all $S \subseteq L$ such that $(D \cap A, S)$ forces agreement on $e$ and $(D \cap \bar{A}, L-S)$ forces agreement on $i$. Then $\mathscr{P}$ is a $\Pi_{1}^{0, L}$ class.

Case 1: $\mathscr{P} \neq \varnothing$. By Theorem 2.1, relativized to $L$, there exists $S \in \mathscr{P}$ such that $L \oplus S$ is cone avoiding. In particular, $S$ and $L-S$ are cone avoiding as well.

Subcase 1a: $S$ is infinite. We then set $(\widetilde{D}, \widetilde{L})=(D, S)$, and claim that if $G$ is any set satisfying $(\widetilde{D}, \widetilde{L})$ then $\Phi_{e}^{G \cap A} \neq C_{s}$. Indeed, if $\Phi_{e}^{G \cap A}$ is total then we can, $S$-effectively from $e$ and the canonical index of $D \cap A$, compute $\Phi_{e}^{G \cap A}(x)$ for any $x$, since we have only to search through finite sets $F$ with $D \cap A \subseteq F \subseteq S$ until we 
find one such that $\Phi_{e}^{F}(x) \downarrow$. Since a set satisfies $(\widetilde{D}, \widetilde{L})$ only if its intersection with $A$ satisfies $(D \cap A, S)$, and since the latter forces agreement on $e$, it follows that we will then have $\Phi_{e}^{F}(x)=\Phi_{e}^{G \cap A}(x)$. Thus, our claim follows by virtue of $S$ being cone avoiding.

Subcase 1b: $L-S$ is infinite. We set $(\widetilde{D}, \widetilde{L})=(D, L-S)$ and can argue as above to conclude that $\Phi_{i}^{G \cap \bar{A}} \neq C_{t}$ for any set $G$ satisfying $(\widetilde{D}, \widetilde{L})$.

Case 2: $\mathscr{P}=\varnothing$. Thus $L \cap A \notin \mathscr{P}$, so we have at least one of the following two possibilities:

Subcase 2a: ( $D \cap A, L \cap A)$ does not force agreement on e. Then for some $x \in \omega$, and for some finite set $F$ satisfying $(D \cap A, L \cap A), \Phi_{e}^{F}(x) \neq C_{s}(x)$. We set $\widetilde{D}=D \cup F$, and let $\widetilde{L}$ consist of all elements of $L$ greater than $\max (\widetilde{D})$ and use $\left(\Phi_{e}^{F}(x)\right)$. Then $\widetilde{L}={ }^{*} L$, hence $\widetilde{L}$ is cone avoiding, and clearly $\Phi_{e}^{G \cap A} \neq C_{s}$ for any set $G$ satisfying $(\widetilde{D}, \widetilde{L})$.

Subcase 2b: $(D \cap \bar{A}, L-A)$ does not force agreement on $i$. We can then analogously define $(\widetilde{D}, \widetilde{L})$ so that $\Phi_{e}^{G \cap \bar{A}} \neq C_{t}$ for any set $G$ satisfying $(\widetilde{D}, \widetilde{L})$.

In either case, $(\widetilde{D}, \widetilde{L})$ extends $(D, L)$ and has the desired properties.

Now if $A$ and $C$ are computable in $0^{\prime}$ and $(D, L)$ is low, we can argue as in the proof of the previous lemma. Briefly, an index for $\mathscr{P}$ can, since $A$ is $\Delta_{2}^{0}$, be found $0^{\prime}$-effectively from $e, i, s, t$ and an index for $(D, L)$, and from this index, $S$ in Case 1 can be found $0^{\prime}$-effectively so that $S$ and $L-S$ are both low. The distinction between Cases 1 and 2 is $L^{\prime}$-effective, hence $0^{\prime}$-effective, from an index for $\mathscr{P}$, while a true subcase of Case 1 (Subcase $1 \mathrm{a}$ or $1 \mathrm{~b}$ ) can be chosen $\mathbf{d}$-effectively. To distinguish between Subcases $2 \mathrm{a}$ and $2 \mathrm{~b}$, we can, since $A, C_{s}, C_{t} \leq_{T} 0^{\prime}$, search $0^{\prime}$ effectively through $\omega$ and all finite sets until we find some $x \in \omega$ and either a finite set $F$ satisfying $(D \cap A, L \cap A)$ with $\Phi_{e}^{F}(x) \downarrow \neq C_{s}(x)$, or a finite set $F$ satisfying $(D \cap \bar{A}, L-A)$ with $\Phi_{i}^{F}(x) \downarrow \neq C_{t}(x)$.

Lemma 4.8. Let $A$ be any set, $(D, L)$ a cone avoiding condition, and $e, i, s, t \in \omega$. (i) There is a cone avoiding extension of $(D, L)$ such that either $(G \cap A)^{\prime}(e)$ is the same for all sets $G$ satisfying this extension, or $\Phi_{i}^{G \cap \bar{A}} \neq C_{t}$ for all such $G$.

(ii) There is a cone avoiding extension of $(D, L)$ such that either $(G \cap \bar{A})^{\prime}(i)$ is the same for all sets $G$ satisfying this extension, or $\Phi_{e}^{G \cap A} \neq C_{s}$ for all such $G$.

If, additionally, $A, C \leq_{T} 0^{\prime}$ and $(D, L)$ is low, then $(\widetilde{D}, \widetilde{L})$ can be chosen low, and its index can be found $\mathbf{d}$-effectively from $e, i$, and an index for $(D, L)$.

Proof. We prove (i), the proof of (ii) being similar. Let $\mathscr{P}$ be the $\Pi_{1}^{0, L}$ class of all sets $S \subseteq L$ such that $(D \cap A, S)$ forces $e$ out of the jump and $(D \cap \bar{A}, L-S)$ forces agreement on $i$.

Case 1: $\mathscr{P} \neq \varnothing$. By Theorem 2.1, relativized to $L$, there exists $S \in \mathscr{P}$ such that $S$ and $L-S$ are cone avoiding.

Subcase 1a: $S$ is infinite. Set $(\widetilde{D}, \widetilde{L})=(D, S)$. If $G$ satisfies $(\widetilde{D}, \widetilde{L})$, then $e \notin$ $(G \cap A)^{\prime}$ as in Subcase 1a of the proof of Lemma 4.6.

Subcase 1b: $L-S$ is infinite. We set $(\widetilde{D}, \widetilde{L})=(D, L-S)$, concluding that $\Phi_{i}^{G \cap \bar{A}} \neq C_{t}$ for all $G$ satisfying $(\widetilde{D}, \widetilde{L})$, as in Subcase 1b of the proof of Lemma 4.7.

Case 2: $\mathscr{P}=\varnothing$. Then $L \cap A \notin \mathscr{P}$, so at least one of the following must obtain: 
Subcase 2a: some finite set $F$ with $\Phi_{e}^{F}(e) \downarrow$ satisfies $(D \cap A, L \cap A)$. We set $\widetilde{D}=D \cup F$, and let $\widetilde{L}$ consist of all elements of $L$ greater than $\max (\widetilde{D})$ and use $\left(\Phi_{e}^{F}(e)\right)$. As in Subcase 2a of the proof of Lemma 4.6, it follows that $e \in(G \cap A)^{\prime}$ for every set $G$ satisfying $(\widetilde{D}, \widetilde{L})$.

Subcase 2b: $(D \cap \bar{A}, L-A)$ does not force agreement on $i$. Then for some $x \in \omega$, and some finite set $F$ satisfying $(D \cap \bar{A}, L-A), \Phi_{i}^{F}(x) \neq C_{t}(x)$ for any set $G$ satisfying $(\widetilde{D}, \widetilde{L})$.

Now suppose $A$ and $C$ are computable in $0^{\prime}$ and that $(D, L)$ is low. An index for $\mathscr{P}$ can, since $A$ is $\Delta_{2}^{0}$, be found $0^{\prime}$-effectively from $e, i, t$ and an index for $(D, L)$. In Case 1 then, this index can be used to find $S, 0^{\prime}$-effectively, so that $S$ and $L-S$ are both low, in addition to being cone avoiding. The distinction between Cases 1 and 2 is $L^{\prime}$-effective, hence $0^{\prime}$-effective, from an index for $\mathscr{P}$, and one may $\mathbf{d}$-effectively choose a true subcase of Case 1. To distinguish Subcases $2 \mathrm{a}$ and $2 \mathrm{~b}$, we can, since $A, C_{t} \leq_{T} 0^{\prime}$, search $0^{\prime}$-effectively through $\omega$ and all finite sets, until we either find a finite set $F$ satisfying $(D \cap A, L \cap A)$ with $\Phi_{e}^{F}(e) \downarrow$, or else some $x \in \omega$ and some finite set $F$ satisfying $(D \cap \bar{A}, L-A)$ with $\Phi_{i}^{F}(x) \downarrow \neq C_{t}(x)$.

\section{Combining Strategies}

In this section, we combine the forcing strategies from the previous section to prove Lemmas 3.1 and 3.2. Again, let $C_{0}, C_{1}, \ldots$ be a fixed sequence of noncomputable sets with $C=\oplus_{i \in \omega} C_{i}$.

Lemma 5.1. Fix a degree $\mathbf{d} \gg \mathbf{0}^{\prime}$. For every uniformly computable sequence $R_{0}, R_{1}, \ldots$ of sets, there exists a cone avoiding set $G$ which is cohesive for this sequence and whose jump is of degree at most $\mathbf{d} \cup \operatorname{deg}(C)$.

Proof. We force with computable conditions to build a set $G$ which, for all $e, i \in \omega$, meets the requirements

$$
\begin{array}{lll}
\operatorname{Req}_{4 e} & : & |G| \geq e \\
\operatorname{Req}_{4 e+1} & : & G \subseteq^{*} R_{e} \text { or } G \subseteq^{*} \overline{R_{e}} \\
\operatorname{Req}_{4 e+2} & : G^{\prime}(e) \text { is decided in the construction } \\
\operatorname{Req}_{4\langle e, s\rangle+3} & : \Phi_{e}^{G} \neq C_{s} .
\end{array}
$$

Let a computable condition $(D, L)$ and $n \in \omega$ be given. If $n=4 e$ we apply Lemma 4.3(i), if $n=4 e+1$ we apply (ii) with $R=R_{e}$, and if $n=4 e+2$ we apply (iii). If $n=4\langle e, s\rangle+3$ we apply Lemma 4.4 with $C=C_{s}$. By the effectiveness of Lemmas 4.3 and 4.4 , we in any case obtain a computable condition $(\widetilde{D}, \widetilde{L})$ such that any set satisfying this extension meets $\mathrm{Req}_{n}$, and an index for this extension can be found $(\mathbf{d} \cup \operatorname{deg}(C))$-effectively from an index for $(D, L)$.

To complete the proof, let $\left(D_{0}, L_{0}\right)=(\varnothing, \omega)$, and iterate the preceding density construction to $(\mathbf{d} \cup \operatorname{deg}(C))$-effectively build a chain

$$
\left(D_{0}, L_{0}\right) \succ\left(D_{1}, L_{1}\right) \succ \cdots
$$

such that, for any $n \in \omega$, any set satisfying $\left(D_{n+1}, L_{n+1}\right)$ meets $\operatorname{Req}_{n}$. Clearly, $G=\bigcup_{i} D_{i}$ has the desired properties. 
Proof of Lemma 3.1. In view of the closing remark in Section 2, we may fix a cone avoiding degree $\mathbf{a} \gg \mathbf{0}$. The $\{0,1\}$-valued partial computable function

$$
\tilde{p}(x)= \begin{cases}\Phi_{e}(x) & \text { if } \Phi_{e}(x) \downarrow \leq 1 \\ \uparrow & \text { otherwise }\end{cases}
$$

consequently has a total, $\{0,1\}$-valued a-computable extension $p$. Hence, the sequence

where for $i \in \omega$,

$$
\vec{R}=\left\{R_{0}, R_{1}, \ldots\right\}
$$

$$
R_{i}=\{x \in \omega: p(e, x)=1\}
$$

is a uniformly a-computable sequence containing all the computable sets. Since $\operatorname{deg}\left(C_{i}\right) \not \leq \mathbf{a}$ for all $i \in \omega$, we may relativize Lemma 5.1 to a to consequently obtain a cone avoiding r-cohesive set $X$, as desired.

Now suppose $C \leq_{T} 0^{\prime}$. We can choose a so that $\mathbf{a}^{\prime} \leq \mathbf{0}^{\prime} \cup \operatorname{deg}(C)$, meaning in fact that a can be chosen low, and hence such that $\mathbf{d} \gg \mathbf{a}^{\prime}=\mathbf{0}^{\prime}$. It follows, by the effectiveness of Lemma 5.1, that $X$ can be found so that $\operatorname{deg}(X)^{\prime} \leq \mathbf{d} \cup \operatorname{deg}(C)=$ d.

We next present a slightly weaker form of Lemma 3.2, from which the latter will follow by an easy induction.

Lemma 5.2. Fix a degree $\mathbf{d} \gg \mathbf{0}^{\prime}$.

(i) Any set $A$ either contains or is disjoint from an infinite cone avoiding set.

(ii) If $A, C \leq_{T} 0^{\prime}$, then $A$ contains or is disjoint from an infinite cone avoiding set whose jump has degree at most $\mathbf{d}$.

Proof. (i) If there exists a cone avoiding set $S$ such that either $S \cap A$ is finite or $S \cap \bar{A}$ is finite, then we are done. So assume not. We force with cone avoiding conditions $(D, L)$ to construct a set $G$ which meets, for all $e, i, s, t \in \omega$ the requirements

$$
\begin{array}{ll}
\operatorname{Req}_{2 e} & :|G \cap A| \geq e \text { and }|G \cap \bar{A}| \geq e \\
\operatorname{Req}_{2\langle e, i, s, t\rangle+1} & : \quad \Phi_{e}^{G \cap A} \neq C_{s} \text { or } \Phi_{i}^{G \cap \bar{A}} \neq C_{t} .
\end{array}
$$

We claim that these requirements are dense, in the sense that every cone avoiding condition can be extended to another, with the property that any set satisfying the extension meets a prescribed requirement. So let $(D, L)$ be a cone avoiding condition, and fix $n \in \omega$. If $n=2 e$, then by our opening assumption we may apply Lemma 4.5. If $n=2\langle e, i, s, t\rangle+1$ we apply Lemma 4.7. In any case, we obtain a cone avoiding condition $(\widetilde{D}, \widetilde{L}) \prec(D, L)$ such that any set satisfying this condition meets $\operatorname{Req}_{n}$.

We conclude by setting $\left(D_{0}, L_{0}\right)=(\varnothing, \omega)$, and iterating the preceding argument to construct a chain

$$
\left(D_{0}, L_{0}\right) \succ\left(D_{1}, L_{1}\right) \succ \cdots
$$

such that, for any $n$, any set satisfying $\left(D_{n+1}, L_{n+1}\right)$ meets $\operatorname{Req}_{n}$. Taking $G=$ $\bigcup_{i} D_{i}$, we note that $G \cap A$ and $G \cap \bar{A}$ must both be infinite by virtue of our even-numbered requirements. Now for every $e, i, s, t \in \omega$, either $\Phi_{e}^{G \cap A} \neq C_{s}$ or $\Phi_{i}^{G \cap \bar{A}} \neq C_{t}$ must hold, by virtue of the odd-numbered requirements. It follows that either $\Phi_{e}^{G \cap A} \neq C_{s}$ for every $e, s \in \omega$ or else $\Phi_{i}^{G \cap \bar{A}} \neq C_{t}$ for every $i, t \in \omega$, and we let $X$ be $G \cap A$ or $G \cap \bar{A}$, depending on which of these two possibilities is true. Thus, $X$ is an infinite cone avoiding subset of $A$ or $\bar{A}$. 
(ii) Without loss of generality, assume that neither $A$ nor $\bar{A}$ contains a low cone avoiding subset, since otherwise the result is trivial. We proceed as in (i), except that we force with conditions that are low as well as cone avoiding. We renumber the requirements $2 e$ and $2\langle e, i, s, t\rangle+1$ by $5 e$ and $5\langle e, i, s, t\rangle+1$, respectively, and add the requirements

$\operatorname{Req}_{5\langle e, i\rangle+2}:(G \cap A)^{\prime}(e)$ or $(G \cap \bar{A})^{\prime}(i)$ is determined in the construction

$\operatorname{Req}_{5\langle e, i, t\rangle+3}:(G \cap A)^{\prime}(e)$ is determined in the construction or $\Phi_{i}^{G \cap \bar{A}} \neq C_{t}$

$\operatorname{Req}_{5\langle e, i, s\rangle+4}:(G \cap \bar{A})^{\prime}(i)$ is determined in the construction or $\Phi_{e}^{G \cap A} \neq C_{s}$.

We claim that these expanded requirements are $\mathbf{d}$-effectively dense. Given a low cone avoiding condition $(D, L)$, and an $n \in \omega$, we proceed as above for $n=5 e$ and $n=5\langle e, i, s, t\rangle+1$. If $n=5\langle e, i\rangle+2$, we appeal to Lemma 4.6 , while if $n=5\langle e, i, t\rangle+3$ or $n=5\langle e, i, s\rangle+4$, we appeal to Lemma 4.8(i) and (ii), respectively. In any case, therefore, we pass to an extension $(\widetilde{D}, \widetilde{L})$ which is satisfied only by sets meeting Req $_{n}$. Moreover, since $A$ and $C$ are computable in $0^{\prime}$, the effectiveness of Lemmas $4.5,4.6,4.7$, and 4.8 implies that we can find $(\widetilde{D}, \widetilde{L})$ low and cone avoiding, and that we can do so d-effectively.

We set $\left(D_{0}, L_{0}\right)=(\varnothing, \omega)$, and iterate the density construction to d-effectively obtain a chain

$$
\left(D_{0}, L_{0}\right) \succ\left(D_{1}, L_{1}\right) \succ \cdots
$$

with the property that any set satisfying $\left(D_{n+1}, L_{n+1}\right)$ meets $\operatorname{Req}_{n}$. Let $G=\bigcup_{i} D_{i}$. Then by virtue of the requirements congruent to 0 , modulo $5, G \cap A$ and $G \cap \bar{A}$ are both infinite. One of the following cases must now obtain:

Case 1: $\Phi_{e}^{G \cap A} \neq C_{s}$ and $\Phi_{i}^{G \cap \bar{A}} \neq C_{t}$ for every $e, i, s, t \in \omega$, so both $G \cap A$ and $G \cap \bar{A}$ are cone avoiding. By virtue of the requirements congruent to 2, modulo 5 , for every $e, i \in \omega$, either $(G \cap A)^{\prime}(e)$ or $(G \cap \bar{A})^{\prime}(i)$ is determined during the construction. Hence, either $(G \cap A)^{\prime}(e)$ is determined for every $e \in \omega$, or else $(G \cap \bar{A})^{\prime}(i)$ is determined for every $i \in \omega$. In the former case, $(G \cap A)^{\prime}$ has degree at most $\mathbf{d}$, and so we let $X=G \cap A$. In the latter case, let $X=G \cap \bar{A}$.

Case 2: $\Phi_{e}^{G \cap A}=C_{s}$ for some $e, s \in \omega$. The requirements congruent to 1 , modulo 5 , imply that for every $e, i, s, t \in \omega$, either $\Phi_{e}^{G \cap A} \neq C_{s}$ or $\Phi_{i}^{G \cap \bar{A}} \neq C_{t}$ holds. Hence $G \cap \bar{A}$ is cone avoiding. On the other hand, the requirements congruent to 4 , modulo 5 , imply that for every $e, i, s \in \omega$, either $(G \cap \bar{A})^{\prime}(i)$ is determined in the construction or else $\Phi_{e}^{G \cap A} \neq C_{s}$. It follows, therefore, that $\Phi_{i}^{G \cap \bar{A}} \neq C_{t}$ for all $i, t \in \omega$, and that $(G \cap \bar{A})^{\prime}(i)$ is determined in the construction for all $i \in \omega$, so $(G \cap \bar{A})^{\prime}$ has degree at most d. We set $X=G \cap \bar{A}$.

Case 3: $\Phi_{i}^{G \cap \bar{A}}=C_{t}$ for some $i, t \in \omega$. We can argue as in the previous case, but appeal instead to the requirements congruent to 1 and 3 , modulo 5 , and set $X=G \cap A$.

In any case, $X$ is an infinite cone avoiding subset of $A$ or $\bar{A}$ and $X^{\prime}$ is determined in the construction. Since the construction is d-effective, this implies that $\operatorname{deg}(X)^{\prime} \leq$ d, which completes the proof.

Proof of Lemma 3.2. (i) We prove the lemma in relativized form by induction on $k$, the case $k=2$ being simply a statement of Lemma 5.2(i). Since that lemma clearly relativizes, we may assume that the present result, along with all its relativizations, 
holds for some $k \geq 2$. Let $A_{1}, \ldots, A_{k+1}$ be a sequence of disjoint sets covering $\omega$. Applying Lemma 5.2(i) to $A_{k+1}$ yields an infinite cone avoiding set $\widetilde{X}$ either contained in or disjoint from $A_{k+1}$. In the former case, we can take $\widetilde{X}$ itself for the desired, cone avoiding subset. In the latter, we have $\widetilde{X} \subseteq A_{1} \cup \cdots \cup A_{k}$, and so $A_{1} \cap \widetilde{X}, \cdots, A_{k} \cap \widetilde{X}$ is a sequence of $k$ disjoint sets whose union is $\widetilde{X}$. Relativizing our inductive hypothesis to $\widetilde{X}$ yields an infinite cone avoiding subset $X$ of $A_{i} \cap \widetilde{X}$ for some $i \leq k$, whence since $A_{i} \cap \widetilde{X} \subseteq A_{i}$, the induction is complete.

(ii) We again use induction, with the base case, this time, following from Lemma 5.2 (ii) by taking $\mathbf{d}$ there low over $\mathbf{0}^{\prime}$. Thus, assume the result for some $k \geq 2$, and let $A_{1}, \ldots, A_{k+1}$ be a sequence of disjoint $\Delta_{2}^{0}$ sets covering $\omega$. By Lemma 5.2(ii), $A_{k+1}$ contains or disjoint from an infinite cone avoiding set $\tilde{X}$ whose jump is computable in $\mathbf{d}$, hence a set which is low 2 since $\mathbf{d}^{\prime} \leq \mathbf{0}^{\prime \prime}$. If $\widetilde{X} \subseteq A_{k+1}$, the proof is complete. Otherwise, we apply the induction hypothesis relative to $\widetilde{X}$ to the sequence $A_{1} \cap \widetilde{X}, \ldots, A_{k} \cap \widetilde{X}$, finding an infinite cone avoiding subset $X$ of some $A_{i} \cap \widetilde{X}$ such that $X$ is low 2 over $\widetilde{X}$, and hence low $_{2}$.

\section{A Minimal Pair of $\mathrm{Low}_{2}$ Homogeneous Sets}

The goal of this section is to prove the following extension of Theorem 3.5:

Corollary 6.1. Every computable 2-coloring of pairs admits a pair of infinite low homogeneous sets whose degrees form a minimal pair.

Fix a set $B$ satisfying $B^{\prime} \leq_{T} 0^{\prime \prime}$. The corollary obviously follows from Theorem 1.4 and the next theorem, the proof of which will occupy most of the rest of this section.

Theorem 6.2. Every computable 2-coloring of pairs admits an infinite low ho- $^{-}$ mogeneous set whose degree forms a minimal pair with $\operatorname{deg}(B)$.

In our proof of Theorem 3.4(ii) above, we constructed an infinite low $_{2}$ cone avoiding homogeneous set by controlling its first jump to lie below a fixed degree $\gg \mathbf{0}^{\prime}$. We forced with low cone avoiding Mathias conditions and appealed to a variant of the low basis theorem obtained by adding cone avoidance, namely Theorem 2.1. It would seem natural to replace the requirement that our conditions $(D, L)$ be cone avoiding by the requirement that $(\operatorname{deg}(L), \operatorname{deg}(B))$ form a minimal pair. (Indeed, this latter requirement is necessary if $L$ is introreducible, i.e. computable from all of its infinite subsets.) However, it no longer seems possible to require that $L$ be low. (For example, if $B=0^{\prime}$ and $L$ is even $\Delta_{2}^{0}$, it would follow that $L$ is computable, which is too much to ask, since in our proof we need to apply basis theorems for $\Pi_{1}^{0}$ classes to obtain $L$.) The same difficulty persists even if we insist that $B$ be $\operatorname{low}_{2}$, which would be sufficient to obtain Corollary 6.1 . For let $\mathscr{P}$ be the $\Pi_{1}^{0}$ class of all $\{0,1\}$-valued diagonally noncomputable functions. Each member of $\mathscr{P}$ computes a fixed point free function by Lemma 4.1 of Jockusch, Lerman, Soare, and Solovay [4] (cf. Soare [12], Exercise V.5.9 and Remark VII.1.9). If we take for $B$ any $\Delta_{2}^{0}$ element of $\mathscr{P}$, then by Theorem 2 of Kučera [8] no $\Delta_{2}^{0}$, let alone low, element of this class can have degree forming a minimal pair with $\operatorname{deg}(B)$.

Thus we instead prove the above theorem by directly forcing the second jump of the constructed set to be computable in $0^{\prime \prime}$, which we do by suitably extending the techniques of [1], Section 5. As in that article, one main advantage of presenting the first proof, that is, of controlling only the first jump, is that it uses a considerably 
simpler notion of forcing. In our case, of course, that proof also lent itself to both a new argument for Seetapun's theorem and an answer to Question 1.5, of which the following does only the latter.

Our proof of Theorem 6.2 can, as usual, be broken up into an r-cohesive and a stable part, from which the theorem then follows analogously to the way Theorem 3.4 did from Lemmas 3.1 and 3.2. For completeness, we show how this proof goes following the statements of the necessary lemmas.

Lemma 6.3. There exists a low $r$-cohesive set whose degree makes a minimal pair with $B$.

Here we pause to note the following extension of Theorem 2.5 of Jockusch and Stephan [5] on the existence of a low 2 cohesive degree. It follows at once from Lemma 6.3, the result of Jockusch and Stephan just cited, and the fact mentioned above ( $\mathrm{cf}[5]$, Corollary 2.4) that the cohesive and r-cohesive degrees coincide.

Corollary 6.4. There exists a minimal pair of low 2 cohesive degrees.

Lemma 6.5. Any $\Delta_{2}^{0}$ set $A$ contains or is disjoint from an infinite low $w_{2}$ set whose degree forms a minimal pair with $B$.

Proof of Theorem 6.2. Fix a computable 2-coloring of pairs $f$, and by Lemma 6.3, fix also a low 2 r-cohesive set $X$ with $(\operatorname{deg}(X), \operatorname{deg}(B))$ a minimal pair. Define a stable 2-coloring of pairs $g$ by $g(\{s, t\})=f(\{p(s), p(t)\})$ for all $\{s, t\} \in[\omega]^{2}$, where $p$ is the principal function of $X$. By Lemma 3.3(ii), relative to $X$, there exists a $\Delta_{2}^{0, X}$ set $A$ such that, if $S$ is any infinite subset of $A$ or $\bar{A}, X \oplus S$ computes an infinite homogeneous set for $g$. We have $B^{\prime} \leq_{T} 0^{\prime \prime} \leq_{T} X^{\prime \prime}$, so we may relativize Lemma 6.5 to $X$ to conclude that either $A$ or $\bar{A}$ contains an infinite set $S$ such that $(S \oplus X)^{\prime \prime} \leq_{T} X^{\prime \prime} \leq_{T} 0^{\prime \prime}$ and every set Turing reducible to both $S \oplus X$ and $B$ is Turing reducible to $X$. Letting $\widetilde{H}$ be an infinite homogeneous set for $g$ computed by $S$, we find that $H=p(\widetilde{H})$ is an infinite low 2 homogeneous set for $f$. Then $H$ is low2 since $H \leq_{T} X \oplus S$ and $X \oplus S$ is low 2 . Suppose now that $Y$ satisfies $Y \leq_{T} H$ and $Y \leq_{T} B$. Then $Y \leq_{T} S \oplus X$ (as $H \leq_{T} S \oplus X$ ). It follows that $Y \leq_{T} X$ since $Y$ is Turing reducible to both $B$ and $S \oplus X$. Since $Y$ is Turing reducible to both $X$ and $B$, and the degrees of $X$ and $B$ form a minimal pair, it follows that $Y$ is computable, as needed to complete the proof that $\operatorname{deg}(H)$ and $\operatorname{deg}(B)$ form a minimal pair.

The proofs of Lemmas 6.3 and 6.5 are obtained by modifying those in Cholak, Jockusch, and Slaman [1], Section 5, of Theorems 3.3 and 3.6 of the same article. In particular, we change the forcing conditions used, add minimal pair requirements, and modify some versions of "smallness" to take account of minimal pair requirements. The proofs we are modifying are quite involved, and we have not made a large number of changes. Hence, we proceed by assuming the reader is familiar with Section 5 of [1], and instead focus on the differences. Admittedly, this is at the cost of making the proof deceptively short. The interested reader may wish to consult [1], pages 14-26, for an in-depth introduction with a view towards the underlying motivation, and pages 16-22 for the technical details.

6.1. Proving Lemma 6.3. Let $\left\{\pi_{e}(G, \vec{x})\right\}$ be an effective enumeration of the $\Pi_{1}^{0}$ formulas in the displayed variables, and let $\left\{R_{e}\right\}$ be a listing of the computable sets 
such that an index of the characteristic function of $R_{e}$ is $0^{\prime \prime}$-computable from $e$. We seek to construct a set $G$ meeting, for each $e \in \omega$, the following requirements:

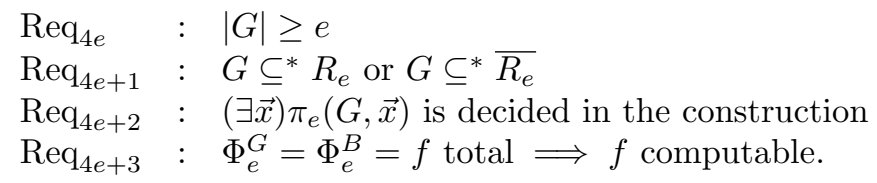

We proceed as in [1], Section 5.1, but instead of forcing with low conditions we will force with those in the following definition. Otherwise, our notation and terminology will be exactly as in [1], Definitions 5.1 and 5.2, and the accompanying discussions.

Definition 6.6. A condition $(D, L)$ is low 2 and minimal pair forming if $L$ is low $_{2}$ and of degree forming a minimal pair with $\operatorname{deg}(B)$. An index for such a condition is a pair of numbers $(d, \ell)$ in which $d$ is the canonical index of $D$ and $\ell$ is a $\Delta_{1}^{0, K^{\prime}}$ index for $L^{\prime \prime}$.

Our main task is to adapt Lemmas 5.3, 5.4, and 5.5 of [1] to the above conditions. The first of these carries over easily. For if $S$ is a finite set of $\Sigma_{2}^{0}$ formulas with at most $G$ free and $\psi(G)$ is a $\Pi_{1}^{0}$ instance of some formula in $S$, then the proof of this lemma actually shows that any $S$-large condition $(D, L)$ can be $L^{\prime}$-effectively, finitely extended to a condition forcing $\neg \psi(G)$. This extension will clearly be low $_{2}$ and minimal pair forming if $(D, L)$ is, in which case it can thus be found $0^{\prime \prime}$ effectively from an index for $(D, L)$ and the Gödel number of $\psi$. Lemma 5.4 of [1] is adapted as follows:

Lemma 6.7. Let a low $w_{2}$ minimal pair forming condition $(D, L)$ of known index be given, along with the canonical index of a finite set $S$ of $\Sigma_{2}^{0}$ formulas free in at most $G$. There is a $0^{\prime \prime}$-effective procedure by which to decide, from these indices, whether or not this condition is $S$-large. If so, there exists an $n \in \omega$ and a sequence $\left(D_{i}, L_{i}, k_{i}, \vec{w}_{i}: i<n\right)$ witnessing this fact such that each $L_{i}$ is low $w_{2}$ and of degree forming a minimal pair with $\operatorname{deg}(B)$. Moreover, this sequence, together with indices for the $\left(D_{i}, L_{i}\right)$, can be found $0^{\prime \prime}$-effectively.

Proof. In the proof of Lemma 5.4 of [1], the definition of $(D, L)$ being $S$-small is written in the form $(\exists z)(\exists Z) P(z, Z, D, L, S)$, where $P$ is a $\Pi_{1}^{0}$ predicate with parameters $Z$ and $L$ of known index (we are identifying the finite sets $D$ and $S$ with their canonical indices). As pointed out there, the predicate $(\exists Z) P(z, Z, D, L, S)$ is consequently $\Pi_{1}^{0, L}$. It follows that, as a predicate of $D$ and $S,(\exists z)(\exists Z) P(z, Z, D, L, S)$ is $\Sigma_{2}^{0, L}$, and we may consequently find a $\Delta_{3}^{0}$ index for it from a $\Delta_{1}^{0, K^{\prime}}$ index for $L^{\prime \prime}$. Hence, from the appropriate indices for $(D, L)$ and $S, 0^{\prime \prime}$ can decide whether or not $(D, L)$ is $S$-small. If it is, we search $0^{\prime \prime}$-effectively for a $z \in \omega$ satisfying $(\exists Z) P(z, Z, D, L, S)$, and then, since the degrees of $L$ and $B$ form a minimal pair, use Theorem 2.5 relative to $L$ to find a solution $Z$ to $P(z, Z, D, L, S)$ which is low $_{2}$ over $L$, hence $\operatorname{low}_{2}$, and whose degree forms a minimal pair with $\operatorname{deg}(B)$. The proof now concludes much like that of Lemma 5.4 of [1], but appealing to the effectiveness of Theorem 2.5 instead of the low basis theorem.

The statement of Lemma 5.5 of [1] now carries over verbatim to $\mathrm{low}_{2}$, minimal pair forming conditions, the proof being identical except of course that all references to Lemma 5.4 of [1] are replaced by references to Lemma 6.7 above. 
Under these modifications, the construction of $G$ can go ahead, mutatis mutandis, as it did in [1], Section 5.1.3. For each $e$, the requirement $s=4 e+3$ is met by applying a modified version of Lemma 4.4 with $C$ in that lemma replaced by $\Phi_{e}^{B}$ to the low 2 , minimal pair forming condition $\left(D_{s-1}, L_{s-1}\right)$ given at stage $s$. (From the proof of Lemma 4.4, it is not necessary to assume that $C$ (now called $\Phi_{e}^{B}$ ) is total. The case divisions are as before, and an oracle for $B^{\prime}$ suffices to find $j$ in Case 1.)

6.2. Proving Lemma 6.5. Let $A$ be a $\Delta_{2}^{0}$ set, and, in keeping with the notation of Cholak, Jockusch, and Slaman [1], page 19, define $A_{0}=A$ and $A_{1}=\bar{A}$. Since we are forcing with conditions that are merely low 2 and not necessarily low, it is not sufficient for our needs to assume that neither $A_{0}$ nor $A_{1}$ has an infinite low subset of degree making a minimal pair with $\operatorname{deg}(B)$. Thus, we assume that neither $A_{0}$ nor $A_{1}$ has even a low 2 such subset, and will construct a subset $G \subseteq A_{i}$ for some $i<2$ to contradict this assumption.

6.2.1. Constructing $G$ inside of $A_{0}$. Following [1], Section 5.2.1, we begin by restricting to low 2 , minimal pair forming conditions $(D, L)$ with $D \subset A_{0}$. We define what it means for a such a condition to be $S$-small 0 or $S$-large ${ }_{0}$ as in [1], page 19. However, in order to ensure that the set $G$ we construct has degree forming a minimal pair with $\operatorname{deg}(B)$, we additionally need the following secondary notion of largeness:

Definition 6.8. Let

$$
S=\left\{(\exists \vec{x}) \psi_{0}(G, \vec{x}), \ldots,(\exists \vec{x}) \psi_{k}(G, \vec{x})\right\}
$$

be a finite set of $\Sigma_{2}^{0}$ formulas free in at most $G$, the $\psi_{i}$ being $\Pi_{1}^{0}$, and let $e \in$ $\omega$. A condition $(D, L)$ is $(S, e)$-small $_{0}$ if there exists an $n \in \omega$ and a sequence $\left(D_{i}, L_{i}, k_{i}, \vec{w}_{i}: i<n\right)$ such that the $L_{i}$ partition $L$, each $k_{i}$ is a number $\leq k$ and each $\vec{w}_{i}$ a tuple of numbers, each $\left(D_{i}, L_{i}\right)$ is a precondition extending $(D, L)$ with $D_{i} \subset A_{0}$, and for each $i<n$, either $\max \left(L_{i}\right) \leq \max \left(\vec{w}_{i}\right)$, or $\left(D_{i}, L_{i}\right)$ forces $\psi_{k_{i}}\left(G, \vec{w}_{i}\right)$, or $\left(D_{i}, L_{i}\right)$ forces agreement on $e$. A condition is $(S, e)-$ large $_{0}$ if it is not $(S, e)$-small $l_{0}$. (This definition is the same as the definition of " $S$-small" except for the final conjunct, which is included for the sake of the eth minimal pair requirement.)

Notice that if a condition is $(S, e)$-large $_{0}$ then it is $S$ - large $_{0}$, and every finite

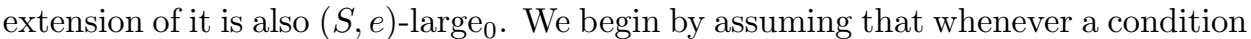

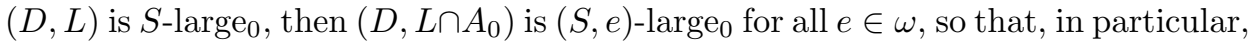

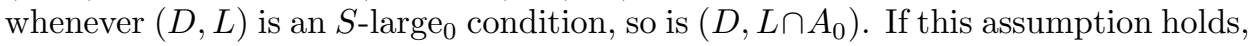
we will construct a suitable $G \subseteq A_{0}$, and otherwise we will construct $G \subseteq A_{1}$. By this assumption, Lemma 5.3 can be adapted to conditions $(D, L)$ with $D \subset A_{0}$ just as in Section 5.2.1 of [1]. The following gives the necessary adaptation of Lemma 5.4 of [1] for our purposes:

Lemma 6.9. Let an index for a low $w_{2}$ minimal pair forming condition $(D, L)$ with $D \subset A_{0}$ be given, along with an $e \in \omega$, and the canonical index of a finite set $S$ of $\Sigma_{2}^{0}$ formulas free in at most $G$. There are $0^{\prime \prime}$-effective procedures by which to decide, from these indices, whether or not this condition is $S$-small $l_{0}$, and, from $e$ and these indices, whether or not it is $(S, e)$-small $l_{0}$. If it is $S$-small or $(S, e)$ small $l_{0}$, there exists an $n \in \omega$ and a sequence $\left(D_{i}, L_{i}, k_{i}, \vec{w}_{i}: i<n\right)$ witnessing this fact such that each $D_{i} \subset A_{0}$, and each $L_{i}$ is low low $_{2}$ and degree forming a minimal 
pair with $\operatorname{deg}(B)$. Moreover, this sequence, together with indices for the $\left(D_{i}, L_{i}\right)$, can be found $0^{\prime \prime}$-effectively.

Proof. In Section 5.2.1 of [1] the definition of $(D, L)$ being $S$-small 0 is put in the form $(\exists z)\left[R^{A}(z) \wedge(\exists Z) P(z, Z, D, L, S)\right]$, where $R^{A}$ is a $\Delta_{1}^{0, A}$ predicate, and $P$ is the same $\Pi_{1}^{0}$ predicate defined in the proof of Lemma 6.7. The definition of $(D, L)$ being $(S, e)$-small 0 can similarly be put in the form $(\exists z)\left[\widetilde{R}^{A}(z) \wedge(\exists Z) \widetilde{P}(z, Z, e, D, L, S)\right]$, where $\widetilde{R}^{A}$ is $\Delta_{2}^{0, A}$ and $\widetilde{P}$ is $\Pi_{1}^{0}$. Thus, $(D, L)$ being $S$-small 0 and $(D, L)$ being $(S, e)$-small 0 can each be defined by some $\Sigma_{1}^{0, A \oplus L^{\prime}}$ predicate, and hence by one computable in $\left(A \oplus L^{\prime}\right)^{\prime}$. But since $A \leq_{T} 0^{\prime} \leq_{T} L^{\prime}$, and since $L$ is low 2 , it follows that $\left(A \oplus L^{\prime}\right)^{\prime} \leq_{T} 0^{\prime \prime}$, and hence that each of these predicates is still $\Delta_{3}^{0}$. The proof can now be completed just as that of Lemma 6.7 .

Now Lemma 5.5 of [1] can be adapted in the obvious way to $\mathrm{low}_{2}$, minimal pair forming conditions $(D, L)$ with $D \subset A_{0}$. Its proof is the same, except that Lemma 6.9 above is used in place of Lemma 5.4 of [1].

Finally, we have the following lemma which will ensure that the set we end up constructing is of degree forming a minimal pair with $\operatorname{deg}(B)$ :

Lemma 6.10. Let $(D, L)$ be a low $w_{2}$ minimal pair forming condition which is $S$ large $_{0}$, and let $e \in \omega$. There exists a low $w_{2}$, minimal, pair forming condition extending $(D, L)$ which is $S$-large $e_{0}$, and which is satisfied only by sets $G$ such that $\Phi_{e}^{G}$ is computable whenever it is total and equal to $\Phi_{e}^{B}$. Furthermore, an index for this extension can be found $0^{\prime \prime}$-effectively from e and an index for $(D, L)$.

Proof. By Lemma 6.9, we can $0^{\prime \prime}$-effectively from $e$ and an index for $(D, L)$ determine whether or not this condition is $(S, e)$-small 0 .

If it is, we can $0^{\prime \prime}$-effectively find an $n \in \omega$ and a sequence $\left(D_{i}, L_{i}, k_{i}, \vec{w}_{i}: i<n\right)$ witnessing this in which each $\left(D_{i}, L_{i}\right)$ is low 2 and minimal pair forming. There must clearly be some $i<n$ so that $L_{i}$ is infinite and $\left(D_{i}, L_{i}\right)$ forces agreement on $e$, else we could easily form a sequence of preconditions to witness that $(D, L)$ is

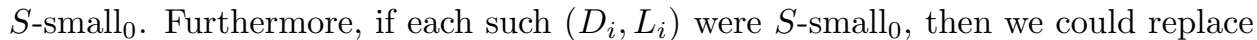
each one by the preconditions from the sequence witnessing this fact, thereby again

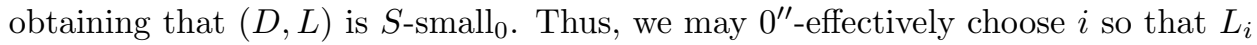
is infinite and $\left(D_{i}, L_{i}\right)$ is $S$-large $_{0}$ and forces agreement on $e$. This condition can serve as the desired extension, since if $G$ satisfies it then $\Phi_{e}^{G}$ is clearly $L$-computable whenever it is total.

If $(D, L)$ is $(S, e)$-large $_{0}$ then by assumption so is $\left(D, L \cap A_{0}\right)$, meaning in particular that it cannot force agreement on $e$. We can thus $0^{\prime \prime}$-effectively find an $x \in \omega$ and a finite set $F$ satisfying $\left(D, L \cap A_{0}\right)$ so that $\Phi^{F}(x) \downarrow \neq \Phi^{B}(x)$. Set $\widetilde{D}=D \cup F$ and let $\widetilde{L}$ consist of all elements of $F$ greater than $\max (F)$ and use $\left(\Phi^{F}(x)\right)$. Clearly, $(\widetilde{D}, \widetilde{L})$ serves as the desired extension.

We now construct an infinite set $G \subseteq A_{0}$ which is low 2 and of degree forming a minimal pair with $\operatorname{deg}(B)$ analogously to the way we constructed an $r$-cohesive such

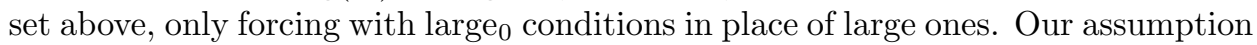
that $A_{1}$ has no low 2 subset of degree forming a minimal pair with $\operatorname{deg}(B)$ ensures that we meet requirement $\mathrm{Req}_{4 e}$ of Section 6.1 above, our adaptations of Lemmas 5.3 and 5.5 of [1] ensure that we meet requirement $\operatorname{Req}_{4 e+2}$, and Lemma 6.10 ensures that we meet $\mathrm{Req}_{4 e+3}$. 
6.2.2. Constructing $G$ inside of $A_{1}$. Assume next that there exists a low 2 , minimal pair forming condition $(\widetilde{D}, \widetilde{L})$, an $\widetilde{e} \in \omega$, and a finite set

$$
\widetilde{S}=\left\{(\exists \vec{x}) \widetilde{\psi}_{0}(G, \vec{x}), \ldots,(\exists \vec{x}) \widetilde{\psi}_{\ell}(G, \vec{x})\right\}
$$

of $\Sigma_{2}^{0}$ formulas free in at most $G$, such that $\widetilde{D} \subset A_{0},(\widetilde{D}, \widetilde{L})$ is $\widetilde{S}$-large lat $_{0}$ but

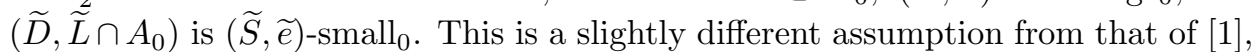
Section 5.2.2, resulting from the fact that we needed Definition 6.8 above in order to obtain Lemma 6.10. Nonetheless, essentially the same argument as there will work here, with only some minor technical alterations along the way. The basic idea is that we switch from trying to build the desired set $G$ inside of $A_{0}$ to building it inside its complement, $A_{1}$.

We need to suitably modify the notion from [1], page 20, of a condition being 1-acceptable, and then that from [1], Definition 5.6, of a condition being $S$-small s. $_{1}$. Here we remark in passing that the latter is misstated in [1], and should, for the purposes of Section 5.2.2 of that article, be defined as in part (iii) of the following definition, but with (5) below not obtaining for any $i<n$.

Definition 6.11. Let a condition $(D, L)$, an $e \in \omega$, and a finite set

$$
S=\left\{(\exists \vec{x}) \psi_{0}(G, \vec{x}), \ldots,(\exists \vec{x}) \psi_{k}(G, \vec{x})\right\}
$$

of $\Sigma_{2}^{0}$ formulas free in at most $G$, be given.

(i) We say $(D, L)$ is 1-acceptable if $D \subset A_{1}, L \subseteq \widetilde{L}$, and $(\widetilde{D}, L)$ is $(\widetilde{S}, \widetilde{e})$-large $_{0}$.

(ii) We say $(D, L)$ is $(S, e)$-small sf $_{1}$ it is 1-acceptable and there exists an $n \in \omega$ and a sequence $\left(D_{i}, L_{i}, k_{i}, \vec{w}_{i}: i<n\right)$ such that the $L_{i}$ partition $L$, each $k_{i}$ is a number $\leq \max (k, \ell)$ and each $\vec{w}_{i}$ a tuple of numbers, each $\left(D_{i}, L_{i}\right)$ is a precondition, and for each $i<n$, at least one of the following holds:

(1) $\max \left(L_{i}\right) \leq \max \left(\vec{w}_{i}\right)$,

(2) $D_{i} \subset A_{1}$, and $\left(D_{i}, L_{i}\right)$ extends $(D, L)$ and forces $\psi_{k_{i}}\left(G, \vec{w}_{i}\right)$,

(3) $D_{i} \subset A_{1}$, and $\left(D_{i}, L_{i}\right)$ extends $(D, L)$ and forces agreement on $e$,

(4) $D_{i} \subset A_{0}$, and $\left(D_{i}, L_{i}\right)$ extends $(\widetilde{D}, \widetilde{L})$ and forces $\widetilde{\psi}_{k_{i}}\left(G, \vec{w}_{i}\right)$,

(5) $D_{i} \subset A_{0}$, and $\left(D_{i}, L_{i}\right)$ extends $(\widetilde{D}, \widetilde{L})$ and forces agreement on $\widetilde{e}$.

(iii) If $(D, L)$ is $(S, e)$-small 1 via a sequence for which (3) above does not obtain for any $i<n$, then we say $(D, L)$ is $S$-small 1 .

(iv) We say $(D, L)$ is $(S, e)$-large $e_{1}$, respectively $S$-large $e_{1}$, if it is not $(S, e)$-small , $_{1}$, respectively if it is not $S$-small 1 .

Any condition which is $(S, e)$-large $_{1}$ obviously enjoys analogs of the two basic properties mentioned following Definition 6.8 for $(S, e)$-large e $_{0}$ conditions. It is also easily seen that if $(D, L)$ is 1-acceptable and finitely extended by $(\widehat{D}, \widehat{L})$ where $\widehat{D} \subset A_{1}$, then this extension is also 1-acceptable. The following analog of Lemma 5.7 of [1] now ensures that we will not at any future stage be forced to switch back to constructing $G$ inside of $A_{0}$.

Lemma 6.12. Let $S$ be a finite set of $\Sigma_{2}^{0}$ formulas free in at most $G$, and let $e \in \omega$. If $(D, L)$ is $S$-large $_{1}$, then so is $\left(D, L \cap A_{1}\right)$. Likewise, if $(D, E)$ is $(S, e)$-large $_{1}$, then so is $\left(D, L \cap A_{1}\right)$.

Proof. By assumption, $\left(\widetilde{D}, \widetilde{L} \cap A_{0}\right)$ is $(\widetilde{S}, \widetilde{e})$ - small $_{0}$, so fix a sequence witnessing this fact. By comparing Definitions 6.8 and 6.11, it is easy to see that this sequence witnesses that $\left(D, L \cap A_{0}\right)$ is $S$-small 1 for any $S$, and hence $(S, e)$-small 1 for any 
$S$ and $e$. This sequence could consequently be combined with any sequence witnessing that $\left(D, L \cap A_{1}\right)$ is $S$-small or $_{1}(S, e)$-small $\operatorname{so~}_{1}$ witness that $(D, L)$ is itself, respectively, $S$-small 1 or $(S, e)$-small 1 .

We can thus adapt Lemma 5.3 of [1] to 1-acceptable, low $_{2}$, minimal pair forming conditions $(D, L)$ with $D \subset A_{1}$ almost exactly as we adapted it in Section 6.2.1 to conditions with $D \subset A_{0}$. Similarly, Lemma 6.9 carries over simply by replacing all instances of $A_{0}$, small $_{0}$ and large by $_{1}$, small ${ }_{1}$ and large ${ }_{1}$, respectively. This leaves Lemma 5.5 of [1] and Lemma 6.10, the proofs of which require a minor case analysis in order to work for 1-acceptable conditions. This is essentially what is required to adapt the former lemma in Section 5.2.2 of [1], but since it is not explicitly described there, we provide the details. The latter lemma can be handled similarly.

Lemma 6.13. Let $(D, L)$ be a low , minimal pair forming condition which is 1-

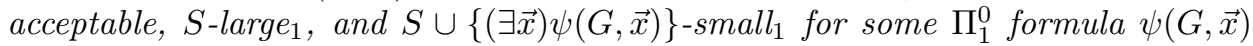
free in at most $G$ and $\vec{x}$. There exists a low $w_{2}$ minimal pair forming extension of this condition which is 1-acceptable and $S$-large ${ }_{1}$, and which forces a $\Pi_{1}^{0}$ instance of $\psi(G, \vec{x})$. An index of this extension can be found $0^{\prime \prime}$-effectively from an index for $(D, L)$, the canonical index for $S$, and the Gödel number of $\psi$.

Proof. Fix $n \in \omega$ and a sequence $\left(D_{i}, L_{i}, k_{i}, \vec{w}_{i}: i<n\right)$ with each $\left(D_{i}, L_{i}\right)$ low $_{2}$ and minimal pair forming to witness that $(D, L)$ is $S \cup\{(\exists \vec{x}) \psi(G, \vec{x})\}$-small ${ }_{1}$. First, notice that if no $\left(D_{i}, L_{i}\right)$ with $L_{i}$ infinite had $D_{i} \subset A_{1}$ then our sequence would in fact witness that $(D, L)$ is $\widehat{S}$-small 1 for any set $\widehat{S}$, and hence in particular that it is $S$-small ${ }_{1}$. Now let $I_{0}$ consist of all $i<n$ such that $L_{i}$ is infinite and $\left(D_{i}, L_{i}\right)$ is 1-acceptable, noting that this must be non-empty else each condition $\left(D_{i}, L_{i}\right)$ with $L_{i}$ infinite and $D_{i} \subset A_{1}$ could be replaced in our sequence by preconditions from

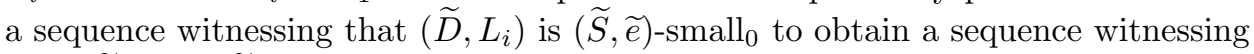
that $(\widetilde{D}, L)$ is $(\widetilde{S}, \widetilde{e})$-small s $_{0}$ and hence that $(D, L)$ is not 1- acceptable. Clearly, each $\left(D_{i}, L_{i}\right)$ with $i \in I_{0}$ forces a $\Pi_{1}^{0}$ instance of some formula in $S \cup\{(\exists \vec{x}) \psi(G, \vec{x})\}$. Let $I_{1}$ consist of all $i \in I_{0}$ with $\left(D_{i}, L_{i}\right) S$-large ${ }_{1}$. If $I_{1}$ were empty, then, depending on whether $i$ is or is not an element of $I_{0}$, each $\left(D_{i}, L_{i}\right)$ with $D_{i} \subset A_{1}$ could be replaced in our original sequence either by preconditions from a sequence witnessing that $\left(\widetilde{D}, L_{i}\right)$ is $S$-small ${ }_{0}$, or else by preconditions from a sequence witnessing that $\left(D_{i}, L_{i}\right)$ is $S$-small 1 , yielding a sequence witnessing that $(D, L)$ is $S$-small 1 . By the effectiveness of Lemma 6.9, we may therefore $0^{\prime \prime}$-effectively from the appropriate indices find an $i \in I_{1}$. Since $\left(D_{i}, L_{i}\right)$ is $S$-large lan $_{1}$, it cannot force a $\Pi_{1}^{0}$ instance of any formula in $S$, and hence it must force $\psi\left(G, \vec{w}_{i}\right)$.

All the lemmas are now combined in the usual way to build the desired $G \subseteq A_{1}$, but forcing with low $_{2}$, minimal pair forming conditions which are 1-acceptable and large $_{1}$, starting with the $\varnothing$-large 1 condition $(\varnothing, \widetilde{L})$.

\section{Questions}

On a visit to Chicago during the preparation of this article, Stephen Simpson asked us the following interesting questions pertaining to Theorem 3.5 and Corollary 6.1 . 
Question 7.1 (Simpson). Given two noncomputable sets $C_{0}$ and $C_{1}$, does every computable 2-coloring of pairs admit an infinite low $_{2}$ homogeneous set $H$ that is cone avoiding for both?

Question 7.2 (Simpson). Does every computable 2-coloring of pairs admit a pair of infinite homogeneous sets whose degrees form a minimal pair and whose join is $\mathrm{low}_{2}$ ?

In response to the first of these, we have the following strengthening of Theorem 3.5:

Theorem 7.3. Let $C_{0}, \ldots, C_{n}$ be a finite sequence of noncomputable sets. Every

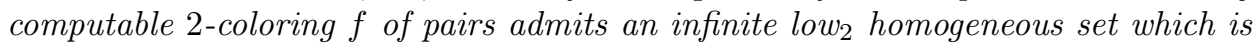
cone avoiding for this sequence.

Proof. Write $\{0, \ldots, n\}$ as a disjoint union $I \cup J \cup K$, where $C_{i} \leq_{T} 0^{\prime}$ for all $i \in I$, $C_{j} \not_{T} 0^{\prime}$ and $C_{j} \leq_{T} 0^{\prime \prime}$ for $j \in J$, and $C_{k} \not_{T} 0^{\prime \prime}$ for $k \in K$. By Theorem 2.1 , relativized to $0^{\prime}$ and applied to the $0^{\prime}$-computable tree all of whose paths have degree $\gg \mathbf{0}^{\prime}$, we can find a degree $\mathbf{d} \gg \mathbf{0}^{\prime}$ such that $\mathbf{d}^{\prime} \leq \mathbf{0}^{\prime \prime}$ and $\operatorname{deg}\left(C_{j}\right) \not \leq \mathbf{d}$ for all $j \in J$ (if $J=\varnothing$, let $\mathbf{d}$ be any degree $\gg \mathbf{0}^{\prime}$ which is low over $\mathbf{0}^{\prime}$ ). Now by Theorem 3.4(ii), there exists an infinite homogeneous set $H$ for $f$ such that $\operatorname{deg}(H)^{\prime} \leq \mathbf{d}$ and $C_{i} \not_{T} H$ for all $i \in I$ (if $I=\varnothing$, let $H$ be any infinite homogeneous set with jump of degree at most $\mathbf{d}$ ). Since $H$ is $\operatorname{low}_{2}$ we also have $C_{k} \mathbb{Z}_{T} H$ for all $k \in K$, as wanted.

We do not know the answer to Question 7.2. A natural attempt at obtaining an affirmative answer is to assume that the set $B$ in Section 6 is low $_{2}$, rather than merely that $B^{\prime} \leq_{T} 0^{\prime \prime}$, and then to try to show that the homogeneous set asserted to exist by Theorem 6.2 can be made to satisfy $(H \oplus B)^{\prime \prime} \leq_{T} 0^{\prime \prime}$. Our obstacle to doing this is that we do not know how to strengthen Theorem 2.5 from asserting the existence of a low 2 path to asserting the existence of one whose join with $B$ is $\operatorname{low}_{2}$.

As already mentioned at the end of Section 3, we do not know the answer to the next question.

Question 7.4. Given a noncomputable set $C$, does every computable 2-coloring of pairs admit an infinite homogeneous set $H \Varangle_{T} C$ whose jump is of degree at most $\mathbf{d}$, where $\mathbf{d}$ is a given degree $\gg \mathbf{0}^{\prime}$ ?

In a similar vein, we can ask the following:

Question 7.5. Given a degree $\mathbf{d} \gg \mathbf{0}^{\prime}$, does every computable 2-coloring of pairs admit a pair of infinite homogeneous sets whose degrees form a minimal pair, and whose jumps are each of degree at most $\mathbf{d}$ ?

In Section 6 we control the second jump of the constructed set, and so it does not appear that this method would be useful in finding an affirmative answer to either of the above questions. But it does not appear that the methods of Sections 4 and 5 could be easily adapted to give affirmative answers either. If we tried to do so for the first question, we would certainly need a different case analysis in the proof of Theorem 3.5, but this would in turn demand a different proof of Theorem 2.1. As for the second question, we would seem to need a version of Theorem 2.5 which is effective in a given degree $\gg \mathbf{0}^{\prime}$, but we do not know of a proof of this theorem which does not make essential use of a $0^{\prime \prime}$ oracle. 


\section{REFERENCES}

[1] Peter A. Cholak, Carl G. Jockusch, Jr., and Theodore A. Slaman, On the strength of Ramsey's theorem for pairs, J. Symbolic Logic 66 (2001), no. 1, 1-55.

[2] Denis R. Hirschfeldt, Carl G. Jockusch, Jr., Bjørn Kjos-Hanssen, Steffen Lempp, and Theodore A. Slaman, The strength of some combinatorial principles related to Ramsey's theorem for pairs, pp. 143-161 in Computational Prospects of Infinity, Part II: Presented Talks, Lecture Notes Series, Institute for Mathematical Sciences, National University of Singapore, Vol. 15, edited by C.T. Chong, Q. Feng, T. Slaman, H. Woodin, and Y. Yang, published by World Scientific, New Jersey, London, Singapore, Beijing, Shanghai, Hong Kong, Taipei, Chennai, 2008.

[3] Tamara Lakins Hummel, Effective versions of Ramsey's theorem: avoiding the cone above 0', J. Symbolic Logic 59 (1994), no. 4, 1301-1325.

[4] Carl G. Jockusch, Jr., Manuel Lerman, Robert I. Soare, and Robert M. Solovay, Recursively enumerable sets modulo iterated jumps and extensions of Arslanov's completeness criterion, J. Symbolic Logic 54 (1989), no. 4, 1288-1323.

[5] Carl G. Jockusch, Jr. and Frank Stephan, A cohesive set which is not high, Math. Logic Quart. 39 (1993), no. 4, 515-530.

[6] Carl G. Jockusch, Jr., Ramsey's theorem and recursion theory, J. Symbolic Logic 37 (1972), no. $2,268-280$.

[7] Carl G. Jockusch, Jr. and Robert I. Soare, $\Pi_{1}^{0}$ classes and degrees of theories, Trans. Amer. Math. Soc. 173 (1972), 33-56.

[8] Antonín Kučera, An alternative, priority-free solution to Post's problem, Mathematical foundations of computer science, 1986 (Bratislava, 1986), Lecture Notes in Comput. Sci., vol. 233, Springer, Berlin, 1986, pp. 493-500.

[9] David Seetapun and Theodore A. Slaman, On the strength of Ramsey's theorem, Notre Dame J. Formal Logic 36 (1995), no. 4, 570-582, Special Issue: Models of arithmetic.

[10] Stephen G. Simpson, Degrees of unsolvability: a survey of results, Handbook of Mathematical Logic (J. Barwise, ed.), North-Holland, Amsterdam, 1977, pp. 631-652.

[11] Stephen G. Simpson, Subsystems of second order arithmetic, Perspectives in Mathematical Logic, Springer-Verlag, Berlin, 1999.

[12] Robert I. Soare, Recursively enumerable sets and degrees, Perspectives in Mathematical Logic, Springer-Verlag, Berlin, 1987, A study of computable functions and computably generated sets.

UNIVERSITY OF CHICAGO

E-mail address: damir@math.uchicago.edu

University of Illinois at URbana-Champaign

E-mail address: jockusch@math.uiuc.edu 